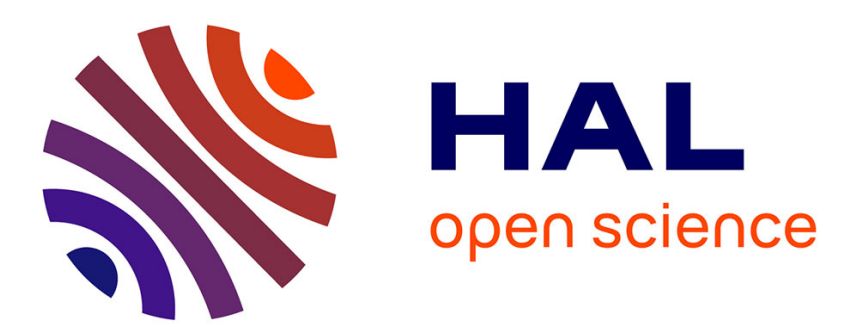

\title{
Experimental analysis and modeling of the losses in the tip leakage flow of an isolated, non-rotating blade setup
}

Benjamin Deveaux, Camille Fournis, Vincent Brion, Julien Marty, Antoine

Dazin

\section{- To cite this version:}

Benjamin Deveaux, Camille Fournis, Vincent Brion, Julien Marty, Antoine Dazin. Experimental analysis and modeling of the losses in the tip leakage flow of an isolated, non-rotating blade setup. Experiments in Fluids, 2020, 61 (126), pp.1-23. 10.1007/s00348-020-02957-z • hal-02909760

\section{HAL Id: hal-02909760 \\ https://hal.science/hal-02909760}

Submitted on 31 Jul 2020

HAL is a multi-disciplinary open access archive for the deposit and dissemination of scientific research documents, whether they are published or not. The documents may come from teaching and research institutions in France or abroad, or from public or private research centers.
L'archive ouverte pluridisciplinaire HAL, est destinée au dépôt et à la diffusion de documents scientifiques de niveau recherche, publiés ou non, émanant des établissements d'enseignement et de recherche français ou étrangers, des laboratoires publics ou privés. 


\title{
Experimental analysis and modelling of the losses in the tip leakage flow of an isolated, non-rotating blade setup
}

\author{
B. Deveaux · C. Fournis · V. Brion · J. Marty · A. Dazin
}

Received: 4 September 2019. Accepted: 11 April 2020

\begin{abstract}
Improving pressure rise capabilities of axial compressors requires an in-depth understanding of the losses produced in the tip leakage region. Here, a generic setup that magnifies the tip region of an isolated, non-rotating blade is used with the objectives of describing the main flow components and evaluating the related sources of loss. The flow at the tip is structured by the jet flow out of the gap which, under the effect of the main stream, rolls-up into a tip-leakage vortex. The current setup is characterized by the tip gap height and the thickness of the incoming boundary layer at the casing, here a flat plate, for a given incidence of the blade. Measurements are performed using $L D V$ and a multi-port pressure probe. Variations in the tip-leakage flow are found to be mainly driven by gap height. A small, intermediate and large gap regimes are more specifically found, with threshold around 4\% and $8 \%$ of gap to chord ratio for the present setting. The incoming boundary layer thickness is shown to provoke a notable effect on the vortex lateral position and total pressure losses. The local entropy creation rate is computed from LDV data and used to identify the sources of loss in the flow. A decomposition into wake and vortex losses is further proposed, allowing to relate the contributions of the various flow components to the overall losses. An empirical model of the formation of the tip vortex is developed to account for the increased losses as a function of gap height. The model provides
\end{abstract}

B. Deveaux · C. Fournis · V. Brion · J. Marty

DAAA, ONERA, Meudon, France

E-mail: vincent.brion@onera.fr

A. Dazin

UMR 9014-LMFL-Laboratoire de Mécanique des Fluides de Lille - Kampé de Friet, Centrale Lille, Arts et Metiers ParisTech, ONERA, CNRS, Univ. Lille, 59000, Lille, France.

E-mail: Antoine.Dazin@ensam.eu a useful mean for the practical approximation of the gap sensitivity of pressure losses.

Keywords Tip-leakage · Vortex - Compressor · Entropy $\cdot$ Losses $\cdot$ Model

\section{Nomenclature}

$\alpha$ Angle of attack

$c$ Blade chord

$h$ Gap height

$\delta_{0.99} \quad 0.99 U_{\max }$ thickness

$\delta^{*} \quad$ Displacement thickness

$\theta^{*} \quad$ Momentum thickness

$H^{*}=\delta^{*} / \theta^{*} \quad$ Shape factor

$R e=U_{\infty} c / \nu \quad$ Chord Reynolds number

$R e_{\theta^{*}}=U_{\infty} \theta^{*} / \nu \quad \theta^{*}$ Reynolds number

$M$ Mach number

$\Gamma \quad$ Tip-leakage vortex circulation

$\Gamma$ " Total secondary vorticity

$\left(y_{v}, z_{v}\right)$ Vortex center position

$C_{P t}=\left(P_{t \infty}-P_{t}\right) /\left(\frac{1}{2} \rho U_{\infty}^{2}\right) \quad$ Total pressure loss coeff.

$C_{\text {wake }}=1-u_{x}^{2} / U_{\infty}^{2}$ Wake loss coeff.

$C_{\text {vortex }}=C_{P t}-C_{\text {wake }}$ Vortex loss coeff.

$K_{P}=\left(P_{s}-P_{s \infty}\right) /\left(\frac{1}{2} \rho U_{\infty}^{2}\right) \quad$ Pressure coeff.

$C_{L} \quad$ Airfoil lift coeff.

$\chi_{D}$ Discharge coeff.

$U_{\infty} \quad$ Upstream velocity

$U_{j} \quad$ Gap exit jet velocity

$P$ Pressure

$T$ Temperature

$s$ Entropy per unit of mass

$\rho$ Density

$\mu$ Dynamic viscosity

$\nu$ Kinematic viscosity 
$u_{i} \quad$ Mean velocity

$u_{i}^{\prime} \quad$ Fluctuating velocity

$S_{i j}=\frac{1}{2}\left(\frac{\partial u_{i}}{\partial x_{j}}+\frac{\partial u_{j}}{\partial x_{i}}\right) \quad$ Strain rate tensor

$\tau_{i j}=-<u_{i}^{\prime} u_{j}^{\prime}>$ Reynolds stress tensor

$k=<u_{i}^{\prime} u_{i}^{\prime}>$ Turbulent kinetic energy

${ }^{m}$ Mass-flow average

- $s$ Surface average

- $\infty$ Upstream quantity

- $t$ Total quantity

- $s$ Static quantity

$<\bullet>$ Ensemble average

- Pressure side quantity

- $\quad$ Suction side quantity

$\nabla \bullet \quad$ Nabla operator

LDV Laser Doppler Velocimetry

\section{Introduction}

The need for a cleaner aviation requires the development of lighter, quieter and more fuel efficient turbojet engines. This can be achieved by increasing the pressure rise capabilities of axial compressors and by extending their range of stable operation. A major obstacle to proceed along this path though lies in the flow going through the gap between the tip of rotor blades and the casing wall of the engine, also known as tip-leakage flow or tip-gap flow. Indeed, the flow at the gap exit rollsup into a tip-leakage vortex that concentrates high total pressure losses (Doukelis et al., 1998a; Kang and Hirsch, 1993) which limit both the compression ratio and the efficiency of compressors. Several studies (Saathoff and Stark, 2000; Vo et al., 2008) also indicate that the impact of the tip-leakage vortex trajectory with the leading edge of the adjacent blade is a precursor of compressor stall.

The first parameter of interest concerning the tip flow phenomenology is gap height. Indeed, while a design parameter of a machine, it is also subject to variations in operation because of wear and thermal loads. That is why knowing its influence on the tip-leakage flow is essential. Previous works have shown that larger gaps lead to higher total pressure losses (Flachsbart, 1931; Lakshminarayana and Horlock, 1963; Storer and Cumpsty, 1994; Doukelis et al., 1998a) and reduce the pressure rise capabilities and the surge margin of compressors (Saathoff, 2001; Kameier and Neise, 1997; Smith and Cumpsty, 1982). However, most of these studies focus on a global analysis of total pressure losses and give little information on the underlying mechanisms of loss generation. Identifying them is hence one of the main motivations of the present paper.
The second parameter of interest is the boundary layer thickness at the casing wall. A computational analysis of its influence on the tip-leakage flow in a compressor rotor and a cascade was performed by Brandt et al. (2002), yielding that a thicker inlet boundary layer leads to higher total pressure losses. Similar results were obtained experimentally by Storer and Cumpsty (1994). In addition, when the boundary layer thickness is increased, not only the tip-leakage vortex detaches from the blade closer to its leading edge but its trajectory also yields more inclination about the blade passage. The interaction of the incoming boundary layer with the blade leading edge is also known to possibly generate a horseshoe vortex. Hot-wire measurements in a compressor rotor performed by Inoue and Kuroumaru (1989) suggested the presence of this structure in the flow. Friction lines visualizations of Kang and Hirsch (1993), in which a saddle point appears at the casing for a small gap height, give credits to this conjecture. Nonetheless, it seems that this tip-leakage flow feature has not been further investigated so far.

Analytical models are often used in preliminary designs of compressors in order to quickly estimate the losses induced by the tip-leakage flow. There is essentially two approaches. The first one, introduced by Prandtl (1919), is based on the lifting line theory which gives an estimation of the vortex induced drag. The presence of the wall, where a slip condition is applied, is materialized by the symmetry plane of a slotted wing. This approach fails to model the tip-leakage flow for small gap heights that represent the biggest interest for turbomachine applications. Indeed, Flachsbart (1931) has shown that induced drag predicted with this approach was overestimated by $30 \%$. To circumvent this problem, Lakshminarayana and Horlock (1963) introduced an empirical coefficient in this model that represents the retained lift at the blade tip. The second approach, initiated by Rains (1954), assimilates the tipleakage flow as a jet in cross-flow. For instance, Denton (1993) with Storer and Cumpsty (1994) built a simple model based on the assumption that the jet flow exiting the gap completely mixes-out with the main flow. Their model gives a good estimation of the increment of total pressure losses with gap height. This second approach, based on an analogy with a jet in cross-flow, seems to be well suited to model the tip-leakage flow and will be considered in the present paper to estimate the circulation and the losses of the tip-leakage vortex.

RANS simulations are widely used in compressor design, insofar as it gives a prediction of the flow for complex geometries with a moderate computational cost. However, because of the complexity of the flow and of the turbulent interactions, the tip-leakage flow 
still represents a challenge for these computations (Kang and Hirsch, 1996; Borello et al., 2007; Decaix et al., 2015). Improvement of the predictions of given by CFD calculations based on RANS approach relies on the availability of detailed experimental data of the tip-leakage flow, as provided here.

The present study uses a generic setup to investigate the flow in the tip region. A single blade is installed in a wind tunnel and set orthogonal to a flat plate that plays the role of the casing wall. This flat and non-rotating setup reproduces many of the important features of the real flow, namely the tip-leakage flow, the tip-leakage vortex and its interaction with the casing wall. Taking these effects alone while discarding the others allows a finer analysis of their characteristics while providing fundamental knowledge, applicable to more realistic situations. This way, conclusions on this generic setup can provide useful guidelines for a wide variety of applications where a tip-leakage flow is present such as compressors, turbines, variable inlet guide vanes and even ducted fans. Furthermore two fundamental parameters of the tip leakage flow, namely gap height and incoming boundary layer thickness at the casing wall, can be easily changed with this generic geometry. The description of the sensitivity of the flow to these two parameters represents the first goal of the work, that naturally offers a phenomenological understanding while exposing its various configurations. The second goal is to identify the sources of total pressure losses and ultimately provide a simple model over the parameter space taken into account. Beyond these goals, the development of a basic experimental database is motivated by the need to provide an improved and extensive collection of data to confront analytical models and CFD calculations.

The present paper is organized in four sections. The experimental setup is presented in more detail in section 2. A detailed description of the effect of gap height and casing boundary layer thickness on different tip-leakage flow features is presented in section 3. The tip-leakage flow losses are analyzed in section 4 and are modelled in section 5 .

\section{Experimental setup}

The data presented here is normalized by the upstream velocity $U_{\infty}$, blade chord $c$ and upstream total temperature $T_{t \infty}$.
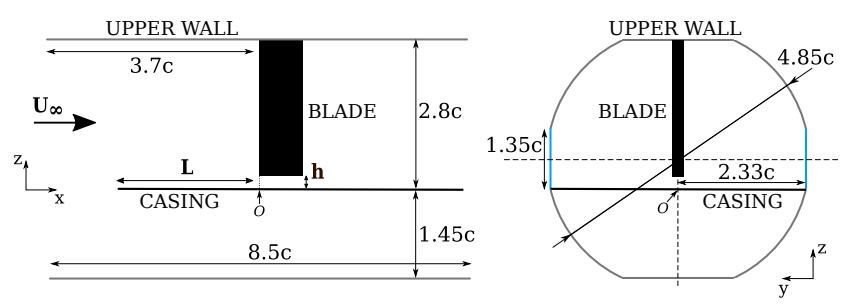

Fig. 1: Schematic view of the isolated blade setup in the test section. $O$ indicates the origin of the coordinate system

Table 1: Experimental parameters

\begin{tabular}{ll}
\hline \hline$U_{\infty}$ & $40 \mathrm{~m} / \mathrm{s}$ \\
$R e$ & 550000 \\
$M$ & 0.1 \\
$T_{u}$ & $0.07 \%$ \\
Airfoil section & $\mathrm{NACA} 0012$ \\
$c$ & $200 \mathrm{~mm}$ \\
$\alpha$ & $10^{\circ}$ \\
& $+0^{\circ}$ for HSV \\
$h / c$ & $0.5 \%$ to $8 \%$ \\
& $+13 \%$ for $L / c=3.3$ \\
& $+0 \%$ and $0.25 \%$ for HSV \\
$L / c$ & $1.8 \quad / 3.3$ \\
\hline \hline
\end{tabular}

\section{Wind tunnel test model}

The analysis is based on wind tunnel tests that have been carried out in the low subsonic S2l facility, located at the ONERA Meudon center. The setup is shown in figure 1. The facility features an open circuit, with a test section of length $2 \mathrm{~m}$ and a circular shape of diameter $1 \mathrm{~m}$. The setup consists of a fixed rectangular wing placed perpendicular to a flat plate, which represents the blade and the casing, respectively. The coordinate system and the origin $O$ are shown in figure 1 .

The parameters considered for this study are provided in table 1 . In order to have the maximum blade loading while preventing flow separation, most of the experiments are conducted with an angle of attack $\alpha$ of $10^{\circ}$. The case $\alpha=0^{\circ}$ is only considered in the analysis of the horseshoe vortex (HSV) upstream of the blade leading edge. Gap height $h$ is changed by adjusting the wingspan with a precision of $\pm 0.05 \mathrm{~mm}$. The error of parallelism between the blade tip and the casing wall is about $0.06^{\circ}$. This setup can reproduce values of $h / c$ encountered in real compressors, from $0.5 \%$ to $3.0 \%$ of the chord while larger gaps, up to $13 \%$ of chord, can also be tuned. An ensemble of discrete values in this range has been considered. Smaller gap heights, $h / c=0$ 
and $h / c=0.25 \%$, are also considered in the analysis of the horseshoe vortex. The boundary layer thickness upstream of the blade is adjusted by changing the distance $L$ between the leading edges of the casing and the blade. Two values of $L / c$ are considered, 1.8 and 3.3. Due to practical reasons, not all combinations of parameters were investigated.

The flow behaves as incompressible (Mach number is nearly 0.1) and freestream turbulence was determined by hot-wire measurements made $1.5 c$ upstream of the blade leading edge. The turbulence intensity $T_{u}$ is $0.07 \%$.

\section{Description of the boundary layers involved in the setup}

This section indicates the preliminary qualification of the boundary layers at the casing, wing surface and upper wall.

Table 2: Casing wall boundary layer characteristics $0.5 c$ upstream of the blade

\begin{tabular}{lll}
\hline \hline$L / c$ & 1.8 & 3.3 \\
\hline$\delta_{0.99 / c}$ & $2.68 \%$ & $4.95 \%$ \\
$\delta^{*} / c$ & $0.40 \%$ & $0.78 \%$ \\
$\theta^{*} / c$ & $0.33 \%$ & $0.60 \%$ \\
$H^{*}$ & 1.30 & 1.30 \\
$R e_{\theta^{*}}$ & 1800 & 3400 \\
\hline \hline
\end{tabular}

\section{Casing}

The turbulent state of the boundary layer at the casing wall is triggered by a tripping tape applied $15 \mathrm{~mm}$ downstream of the plate leading edge (which follows a Rankine body shape). The tripping tape is a $125 \mu \mathrm{m}$ thick zig-zag turbulator tape with $6 \mathrm{~mm}$ width and $70^{\circ}$ angle of the brand Glasfaser-Flugzeug-Service. Twocomponent LDV measurements, described in the next section, have been used to evaluate the boundary layer. These measurements were realized for $h / c=13 \%$ and $\alpha=0^{\circ}$ to mitigate the pressure gradient imposed by the blade. The properties of the boundary layer $0.5 c$ upstream of the blade leading edge are given in table 2 . For the two values of $L$, the shape factor is 1.3 , indicating a fully turbulent state.

\section{Blade}

The same tripping tape as before is placed at the two sides of the airfoil, $20 \%$ of the chord downstream of the leading edge. The tripping tape has an effect on the pressure side only. At the suction side transition

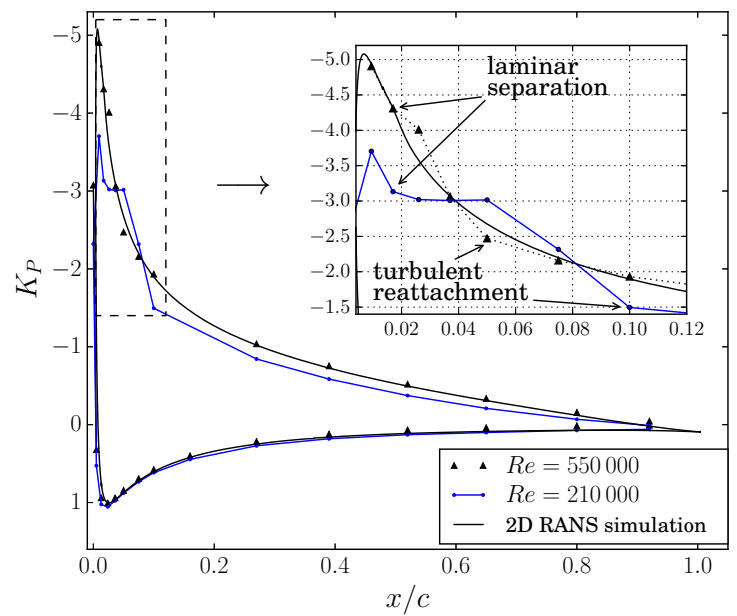

Fig. 2: Pressure distribution around the NACA0012 airfoil for $\alpha=10^{\circ}(h=0)$. Comparison between experimental and numerical, RANS, data at two different Reynolds numbers. The pressure taps in the experiment are located at the altitude $z=1.7 c$

occurs naturally shortly after the leading edge. In figure 2, the measured pressure distribution around the airfoil for a Reynolds number of 550000 is compared to measurements for $R e=210000$ and to a $2 \mathrm{D}$ RANS simulation for $R e=550000$. The calculation uses the fully turbulent $k-\omega S S T$ model of Menter (1994). Figure 2 shows a good agreement between measurements and calculations. The pressure distribution at Reynolds 210000 exhibits a pressure plateau close to the leading edge, which is typical of a laminar separation bubble, see for instance $\mathrm{Hu}$ and Yang (2008). In the insert of figure 2, it can be seen that the pressure distribution for $R e=550000$ also exhibits an alteration of the slope, revealing here also the existence of a short laminar separation bubble, close to the leading edge. Downstream of this laminar separation, due to the destabilization of the bubble, the flow reattaches in a turbulent state, causing the natural laminar-turbulent transition at the suction side, in between $x / c=2 \%$ and $x / c=5 \%$. Due to the fully turbulent flow hypothesis, the calculation does not capture the laminar separation bubble. This explains the small discrepancy between experimental and numerical results, visible in the insert of figure 2 .

\section{Upper wall}

The boundary layer velocity profile at the upper wall (see the description in figure 1) was obtained using a flat Pitot tube placed $2.23 \mathrm{c}$ upstream of the blade leading edge. The boundary layer, displacement and momentum thicknesses are respectively $11.45 \%, 1.45 \%$ and 
$1.15 \%$ of chord. The shape factor is 1.3 , indicating here also a fully turbulent state.

\section{Measurements}

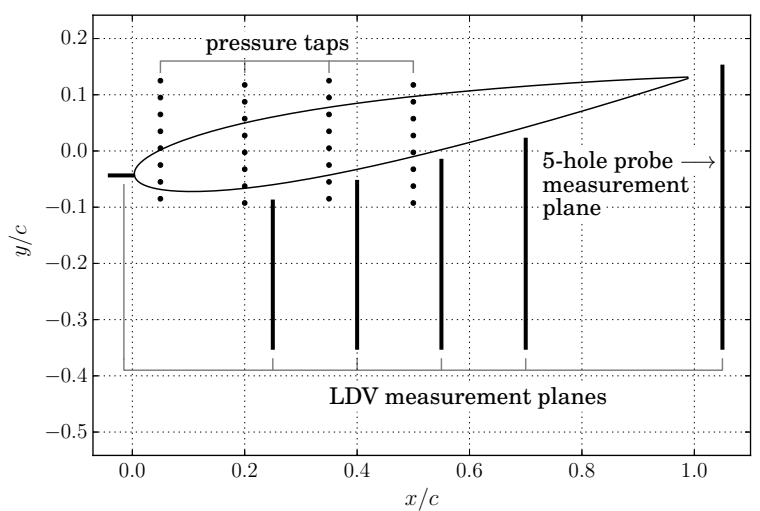

Fig. 3: Description of the various measurements performed in the current experiment. The figure indicates the pressure taps localized at the casing wall in the gap region, the various measurement planes for the LDV and the plane at $x=1.05 \mathrm{c}$ measured using the 5 -hole pressure probe. Mesh resolution is given in table 3

Table 3: Mesh resolution of 5-hole probe and LDV measurement planes

\begin{tabular}{lll}
\hline \hline & $\begin{array}{l}\text { Suction side } \\
(y<0)\end{array}$ & $\begin{array}{l}\text { Leading edge } \\
(x<0)\end{array}$ \\
\hline$\Delta x / c$ & $\varnothing$ & $3.5 \times 10^{-3}$ \\
$\Delta y / c$ & $10 \times 10^{-3}$ & $\varnothing$ \\
$\Delta z / c$ & $5 \times 10^{-3}$ & $0.6 \times 10^{-3}$ \\
\hline \hline
\end{tabular}

The measurement setup is described in figure 3 . The casing is equipped with 32 pressure taps in the gap area, which are connected to an ESP differential pressure scanner. The uncertainty on the pressure measurement is $\pm 16 P a$.

A 5-hole pressure probe was used to measure velocity and pressure in a plane downstream of the blade, as shown in figure 3 . The probe has a diameter of $1.6 \mathrm{~mm}$. The uncertainties associated with its calibration method are \pm 0.001 for the Mach number and $\pm 0.15^{\circ}$ for pitch and yaw angles.

Two-component laser Doppler velocimetry (LDV) was used to investigate the flow at the casing, upstream of the blade and for $\alpha=0^{\circ}$. These measurements were made in forward scattering configuration. The green and blue beams ( $514.5 \mathrm{~nm}$ and $488 \mathrm{~nm}$ ) are conducted to a Dantec Fiberflow emitter. The receiving optics are composed of an in-house Cassegrain telescope $(200 \mathrm{~mm}$ aperture) equipped with two photomultipliers. The spatial resolution is approximately $0.10 \mathrm{~mm}$ and measurements were possible down to approximately $0.15 \mathrm{~mm}$ above the casing. For these measurements the flow is seeded with DEHS droplets generated by a Laskin nozzle and injected upstream of the wind tunnel.

The three-component LDV technique was then employed for $\alpha=10^{\circ}$. The presence of the blade imposed a back scattering configuration for the $(y, z)$ measurement planes as indicated in figure 3 . The optical arrangement for $514.5 \mathrm{~nm}$ and $488 \mathrm{~nm}$ lines is similar to the 2 components configuration. A third pair of laser beams $(532 \mathrm{~nm})$ is conducted to a second emitting optical system and a second Cassegrain telescope is dedicated for receiving the light at this wavelength. In this case the spatial resolution is about $0.15 \mathrm{~mm}$. Because of light reflections it was not possible to get closer than $10 \mathrm{~mm}$ from the blade and $0.3 \mathrm{~mm}$ from the casing in general. In addition other regions of the flow could not be measured, as will be apparent later on. For this configuration a homogeneous seeding at the intake of the wind tunnel is generated with a Magnum fog machine (glycol droplets)

The laser beams are generated by three $1 \mathrm{~W} C o$ herent Genesis laser sources. The Doppler frequency shift is measured with a Dantec $B S A$ processor. The calibration was done by measuring directly the angles of the laser beams with a theodolite. The uncertainty on the velocity measurement coming from the calibration procedure and the optical adjustments is $\pm 0.1 \mathrm{~m} / \mathrm{s}$. The statistical moments are computed on 10000 samples for two-component measurements. For three-component measurements, 3000 samples were taken, which is enough for the estimation of the average to converge and to get a qualitative estimation of the Reynolds stress tensor. The statistical moments are computed with the average weighted by the transit time of the particles in the measurement volume.

\section{Description of the flow}

This section aims at analyzing the sensitivity of the tipleakage flow to the gap height and incoming boundary layer thickness at the casing wall. This analysis is based upon a detailed description of several key features of this flow. 


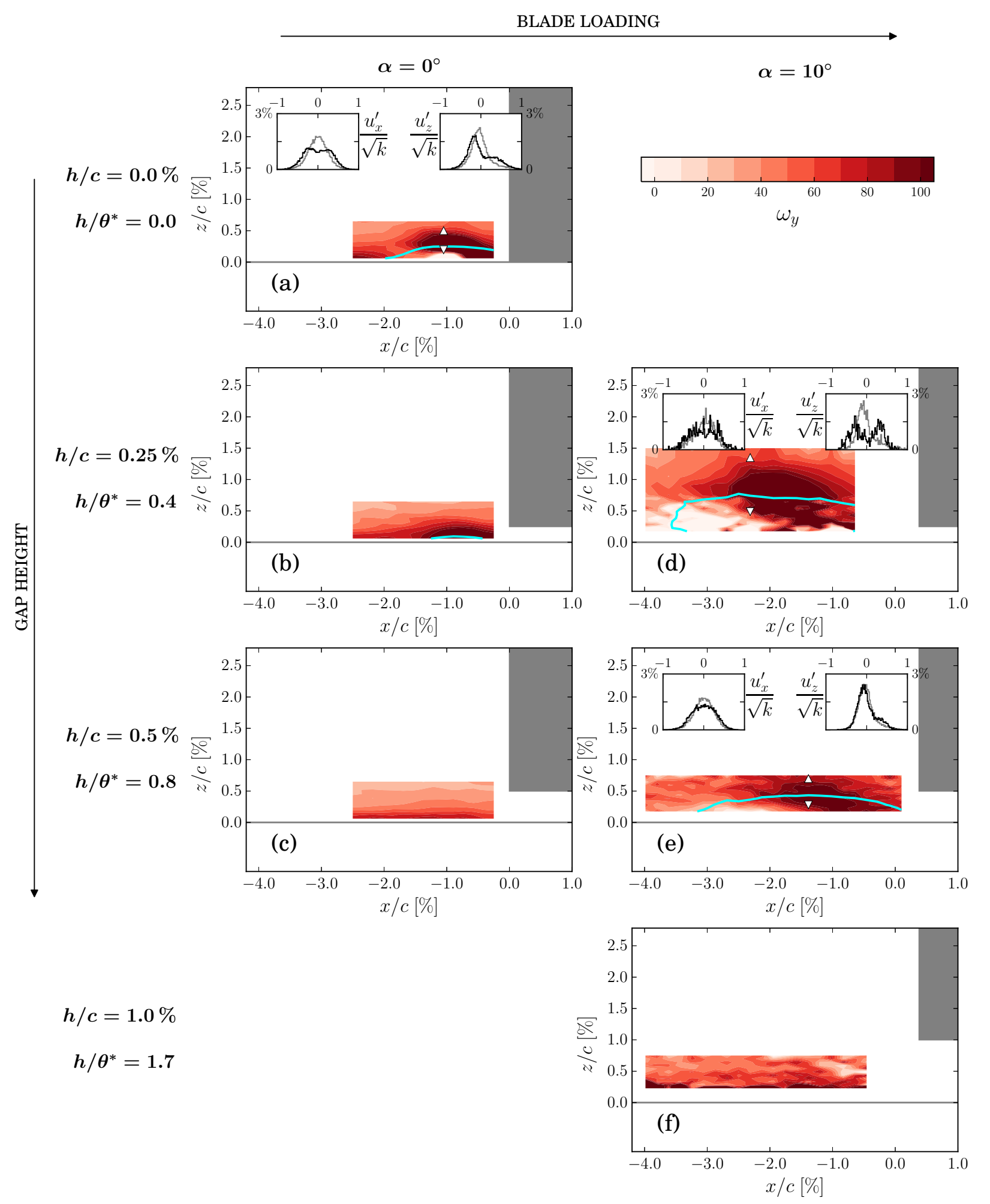

Fig. 4: Description of the flow in the leading edge region. Iso-contours of vorticity $\omega_{y}$ in the leading edge plane. The flow reversal area is delimited by the iso-line of $u_{x}=0$, plotted in blue. The inserts show the pdfs of the velocity fluctuations inside the reversal flow area, at location specified by $(\nabla)$ using black curves and the pdfs of the velocity fluctuations outside the reversal flow area, at location specified by $(\triangle)$ using gray curves 


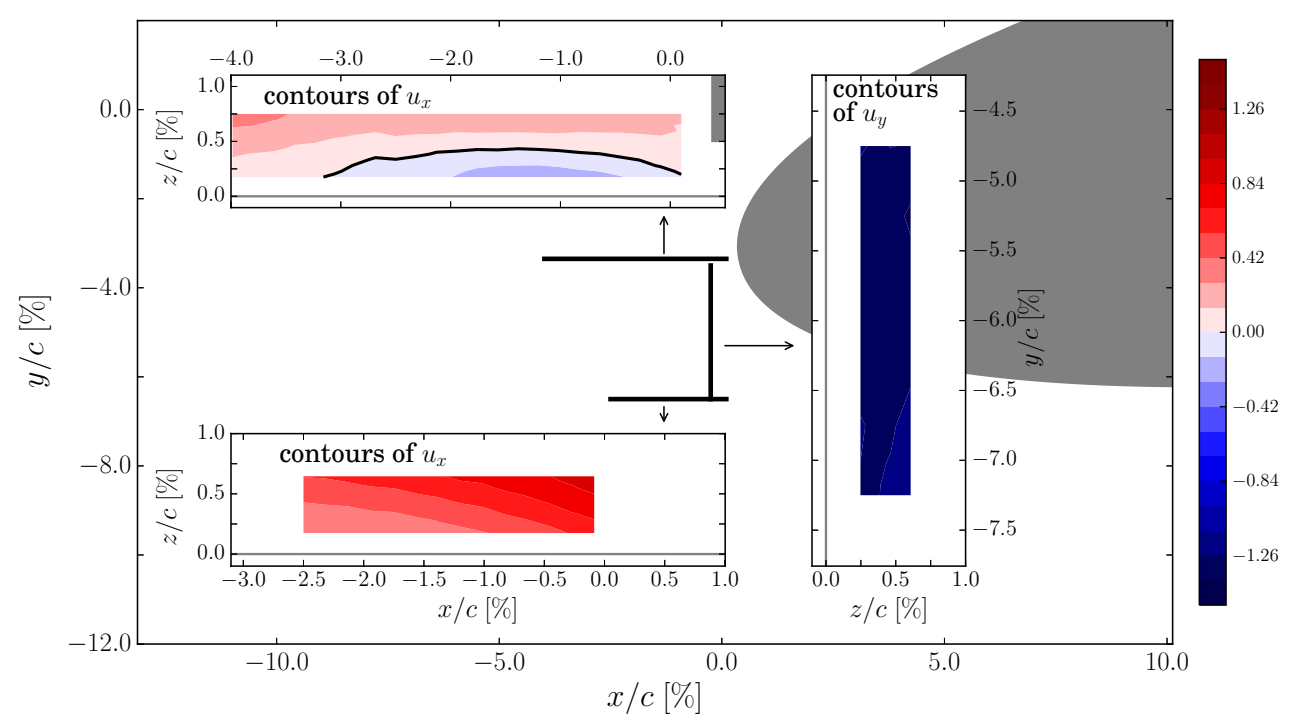

Fig. 5: Velocity field in the leading edge area for $h / c=0.5 \%\left(h / \theta^{*}=0.8\right)$ and $\alpha=10^{\circ}$. The locations of the three planes are indicated at the center of the figure. Inserts on the top and bottom left show contours of $u_{x}$ in $(x, z)$ planes, where the flow essentially goes from left to right. Insert on the right shows contours of $u_{y}$ in a $(y, z)$ plane, where the flow essentially goes from top to bottom of the figure

\section{Horseshoe vortex in the leading edge area}

A horseshoe vortex can form for small gap height as observed, for instance, by Kang and Hirsch (1993). Here the presence of a horseshoe vortex is investigated by looking at the velocity field in a $(x, z)$ plane upstream of the blade leading edge, as shown in figure 3 . The reference situation for a horseshoe vortex is for zero gap height. As explained by Millikan (2018), the formation process relies on the reorientation of the vorticity present in a boundary layer around the object opposing the flow.

Gap height, given in ratio of the incoming boundary layer momentum thickness $h / \theta^{*}$, and blade loading are the fundamental parameters of the problem and are varied thereafter. The thick incoming boundary layer case is considered for varying $h / \theta^{*}$ as it produces larger structures in physical space, which eases measurements. The analysis uses the vorticity field in front of the blade leading edge obtained from LDV. The results are shown in figure 4.

A horseshoe vortex is found in the limit case of a wing-body type junction $h=0$. This is apparent in figure $4 \mathrm{a}$ and agrees with the results by Hasan et al. (1986), Devenport and Simpson (1990) and Bloxham et al. (2008). The flow presents an area of reversal flow close to the casing wall and a region of high vorticity $\omega_{y}$, which is similar to the PIV measurements conducted by Bloxham et al. (2008), upstream of a junction between a cylinder and a flat plate. It also appears that the horseshoe vortex switches between two preferred states over time, as indicated by the probability density functions (pdf) of the flow at several locations about the reversal flow region. Outside this reversal area, at location specified by $(\triangle)$ in figure 4 a, both $u_{x^{-}}^{\prime}$ and $u_{z}^{\prime}$-pdfs have a Gaussian shape. Inside this area, at location specified by $(\nabla)$, the pdf of $u_{x}^{\prime}$ exhibits two maxima and a similar account can be made with the $u_{z}^{\prime}$-pdf, although it features a weaker intensity. This two peaked pdfs behaviour is usually associated with bimodal dynamics, and has been observed in several studies on horseshoe vortices before (Devenport and Simpson, 1990; Yakhot et al., 2006; Praisner and Smith, 2006). It thus appears that bimodality is quite generic of horseshoe vortices. Although a systematic validation of this property is strictly speaking lacking in current state of knowledge, it is helpful to consider it as a marker of the vortex nature of the flow reversal observed in the present flow, as otherwise distinguishing between a simply recirculating flow and a vortex is uneasy.

The conditions for the appearance of a horseshoe vortex at finite gap heights are investigated in the rest of figure 4. The largest gap heights, in figures 4c and 4f, shows no flow reversal, which amounts to the boundary layer remaining attached. A reversal flow area appears when the ratio $h / \theta^{*}$ is reduced below 0.4 for $\alpha=0^{\circ}$ and 0.8 for $\alpha=10^{\circ}$, see figures $4 \mathrm{~b}$ and $4 \mathrm{e}$. With further reduction of $h / \theta^{*}$, the reversal flow and high vortic- 
ity areas widen, see figures $4 \mathrm{a}$ and $4 \mathrm{~d}$. The effect of blade incidence also indicates that increasing the blade loading promotes the apparition of a separation point at the casing wall, thereby increasing the critical gap height for flow separation.

In order to evaluate the presence of a horseshoe vortex for the case $\alpha=10^{\circ}$ and $h / \theta^{*}=0.8$, shown in figure $4 \mathrm{e}$, additional LDV measurements were carried out in vertical planes adjacent to the leading edge and are displayed in figure 5. It can be seen that a region of reversal flow is only visible in the leading edge plane. This indicates that reversal flow is limited to the area upstream of the blade leading edge and furthermore that the reversal flow observed in figure 4e most likely corresponds to a small separation bubble. Moreover figure 4e shows that the $u_{x}^{\prime}$-pdfs inside and outside the reversal flow region are similar and have a Gaussian shape. Even if the $u_{z}^{\prime}$-pdf inside the reversal flow area exhibits another small peak, it does not differ much from the $u_{z^{-}}^{\prime}$ pdf outside of the reversal flow area. Therefore this flow has no perceptible bimodal behaviour. On the basis of previous approach, the flow for $\alpha=10^{\circ}$ and $h / \theta^{*}=0.8$ hence does not seem to form a horseshoe vortex. However for $\alpha=10^{\circ}$ and $h / \theta^{*}=0.4$, shown in figure $4 \mathrm{~d}$, the $u_{z}^{\prime}$-pdf in the reversal flow region exhibits two maxima, revealing the presence of a bimodal dynamics, and suggesting, in this case, the formation of a horseshoe vortex.

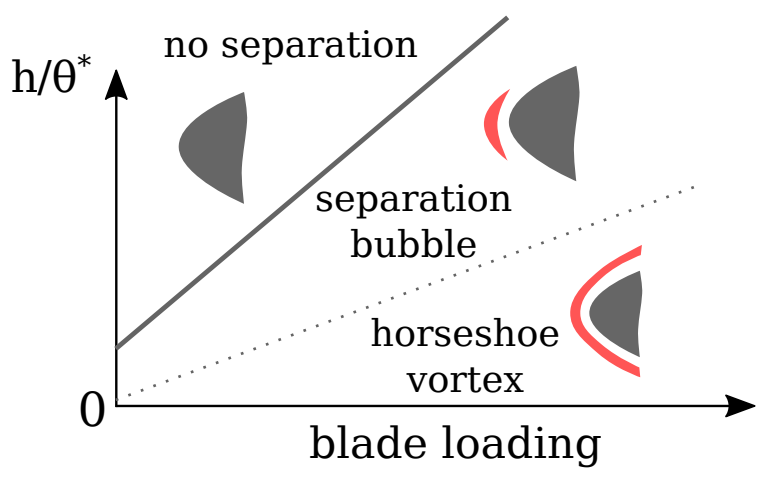

Fig. 6: Schematic of the flow topology at the blade leading edge as a function of blade loading and $h / \theta^{*}$, as derived from the present observations. The thick continuous gray line indicates the threshold $\left(h / \theta^{*}\right)_{s}$.

The sketch in figure 6 summarizes the evolution of the flow topology in front of the blade leading edge with the blade loading and $h / \theta^{*}$ as parameters, based on previous findings. When $h / \theta^{*}$ is large, this sketch states that no separation occurs at the casing wall. When $h / \theta^{*}$ is reduced below a critical value $\left(h / \theta^{*}\right)_{s}$, a separation bubble appears which eventually becomes more intense upon further reduction of $h / \theta^{*}$. The limit case $h / \theta^{*} \rightarrow 0$ corresponds to a junction flow with a horseshoe vortex. A higher blade loading shifts the critical ratio $\left(h / \theta^{*}\right)_{s}$ toward higher values, which is indicated by the positive slopes of the separation lines.

\section{Transverse flow in the gap}

The flow inside the gap sustains many changes important to consider in order to understand the overall tip flow. However from an experimental point of view at least, its qualification is a challenging task, especially regarding flow velocity. Average and RMS values of velocity inside the gap were obtained by Heyes et al. (1992) using single hot-wire measurements in a transverse vertical plane, and by Jacob et al. (2010) with two-component PIV measurements in a horizontal plane parallel to the casing wall. In the present study, the velocity inside the gap is obtained using 3component LDV measurements for a gap height of $6 \%$ of chord and a thick incoming boundary layer at the casing. This gap height is large enough to allow optical measurement and is close to the value of $5 \%$ of chord considered by Jacob et al. (2010). These LDV measurements are shown along with static pressure measurements at the casing wall in figure 7 , for $x / c=40 \%$. The same horizontal axis is used throughout figure 7 to ease interpretation.

The data in figure $7 \mathrm{a}$ first shows that the flow undergoes a strong acceleration from the pressure side toward the gap. The evolution of the maximum transverse velocity along $y$ is plotted in figure $7 \mathrm{~b}$, along with the associated other velocity components. The gap essentially impacts the transverse components $u_{y}$ and $u_{z}$, whereas the streamwise component $u_{x}$ is weakly affected (on the order of $10 \%$ ). This is important as it suggests that the velocity components in the transverse direction can be analyzed independently from the streamwise component, as previously done by Rains (1954). The flow acceleration at the gap entrance generates a pressure drop well captured by the evolution of the static pressure coefficient $K_{P}$ at the casing wall, shown in figure $7 \mathrm{c}$. Note that

$K_{P}=\frac{P_{s}-P_{s \infty}}{\frac{1}{2} \rho U_{\infty}^{2}}$

with $P_{s}$ and $P_{s \infty}$ the local and freestream static pressures, respectively. The $K_{P}$ distribution is shown at $x / c=0.35$ and $x / c=0.50$, displaying little variation in this region of $x / c$. Interestingly, the minimum of static pressure is located at $y / h \simeq-1$, that is at a distance of one gap height from the pressure side, which is comparable to the experimental data of Moore and Tilton 

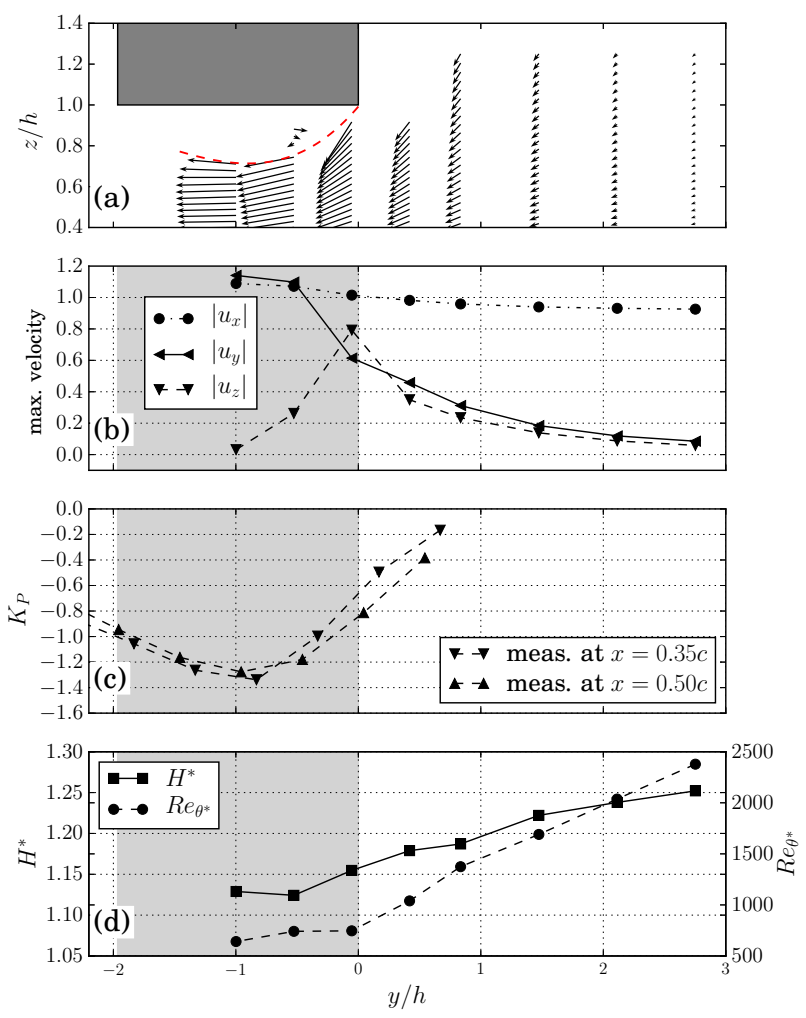

Fig. 7: Topology of the flow entering the gap for $h / c=6.0 \%$ at $x / c=0.40$. (--): approximate boundary of the separation bubble. The origin of the $y$ axis is shifted to the gap entrance. The flow goes from right to left. (a) : Transverse flow using vectors of the transverse velocity. (b) : Evolution of the maximum velocity of each component (axial, transverse, vertical) along the transverse direction. (c) : Evolution of the pressure coefficient along the transverse direction. (d) : Evolution of the shape factor and Reynolds number based on momentum thickness of the streamwise boundary layer as it goes through the gap

(1988) and of Heyes et al. (1992). Moreover, a comparison of figures $7 \mathrm{~b}$ and $7 \mathrm{c}$ indicates that the minimum of static pressure corresponds to the maximum transverse velocity $u_{y}$.

Figure 7a also indicates the formation of a separated flow region at the tip of the blade, as a consequence of its sharp edge. The separated region narrows the effective passage area, which reaches a minimum at a location known as vena-contracta. Comparing figure $7 \mathrm{a}$ and $7 \mathrm{c}$ indicates that the position of the vena-contracta matches the location of the minimum of static pressure at the casing wall, i.e. $y / h \simeq-1$, in coherence with the work of Moore and Tilton (1988).

To analyze the impact of the tip-leakage flow on the streamwise boundary layer at the casing wall, the evolution of the shape factor $H^{*}$ and Reynolds number $R e_{\theta^{*}}$, computed from $u_{x}$ profiles, are plotted in figure 7 d. It can be seen that $H^{*}$ diminishes from 1.25 to 1.13 while $R e_{\theta^{*}}$ decreases from 2300 to 640 in the direction of the vena-contracta. This behaviour is typical of a boundary layer in a favourable pressure gradient, which is imposed by the accelerating flow in the gap, and is consistent with the measurements realized by Julien et al. (1969).

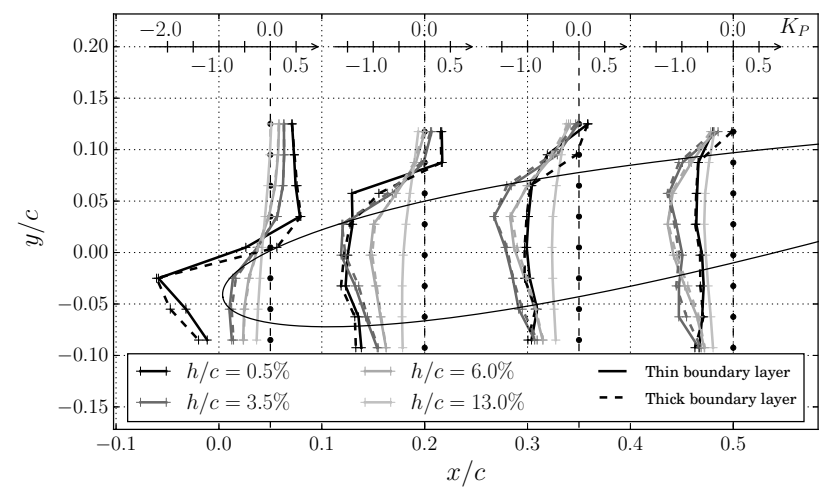

Fig. 8: Transverse distribution of static pressure at the casing wall, in the gap region, for a selection of axial stations and for various gap heights

The other gap sizes were not dedicated to LDV measurements, especially small gaps for which the LDV could not be applied, and are investigated using the pressure distribution available at the casing wall. Figure 8 displays the effect, on this pressure distribution, of gap height $h / c$, considering two incoming boundary layer thicknesses at the casing wall. The transverse evolution of pressure traduces the transverse momentum flowing through the gap as explained before. The main finding concerns the chordwise location of the maximum transverse flow, or minimum static pressure, that is seen to move downstream with increasing gap size. For $h / c=0.5 \%$, the location is near the blade leading edge, while it is around $x / c=0.2$ for $h / c=3.5 \%$ and $x / c=0.35$ for $h / c=6 \%$. The incoming boundary layer thickness has little influence here.

Detailed measurements of the flow at the gap exit were realized by Storer and Cumpsty (1991) with a pressure probe. They provided profiles of total pressure losses and of two components of the velocity in a plane parallel to the casing. Those velocity and total pressure losses profiles show that the flow exiting the gap is similar to a jet. The objective here is to complete this description using 3-component LDV measurements, performed in several planes near the suction side of the blade previously shown in figure 3 . Velocity 


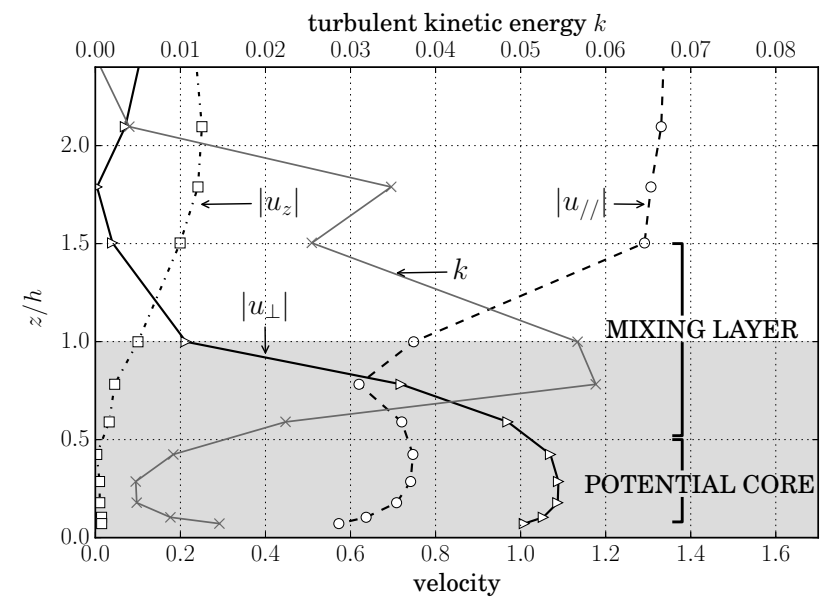

Fig. 9: Velocity and turbulent kinetic energy profiles at the gap exit for $h / c=3.5 \%$ at $(x, y)=(0.55 c,-0.02 c)$. $u_{/ /}$(resp. $u_{\perp}$ ) designates the velocity component parallel (resp. normal) to the chord

and turbulent kinetic energy profiles close to the gap are plotted in figure 9 for $h / c=3.5 \%$ and $x / c=0.55$, where $u_{/ /}$(resp. $u_{\perp}$ ) corresponds to the velocity component parallel (resp. normal) to the chord. The strong transverse flow through the gap induces a jet like flow at the suction side. This can be seen looking at the profiles of the velocity component $u_{\perp}$ and the turbulent kinetic energy $k$. These share similarities with an isolated jet considering for instance details given by Pope (2011). Especially a potential core and a mixing layer are found. The potential core corresponds to the area where $u_{\perp}$ is high and $k$ is low, and the mixing layer can be recognized as the area where $k$ and the gradient of $u_{\perp}$ are high. In addition the transverse flow exits the gap parallel to the casing since the vertical velocity $u_{z}$ is close to zero in the gap area, i.e. for $z / h<1$. Finally, the profile of $u_{/ /}$exhibits a deficit in the mixing layer, which is the expression of the turbulent losses induced in this region. Of course this jet-like flow interacts with the main flow, and this creates a situation best referred to as a jet in cross-flow. The consequence of this interaction is the rolling-up of the jet into a tip-leakage vortex, as described in the next section.

\section{Tip-leakage vortex}

Figure 10 shows iso-lines of the streamwise vorticity field in a transverse plane at $x / c=1.05$, downstream of the blade. The area of negative vorticity corresponds to the tip leakage vortex described previously. The proximity of the casing wall generates an opposite vortical zone, which essentially forms as a consequence of the separation of the transverse boundary layer induced by the tip vortex at the casing wall, as previously observed by Muthanna and Devenport (2004). The effect of gap height and boundary layer thickness on the tip-leakage vortex trajectory, circulation and axial velocity is analyzed thereafter.

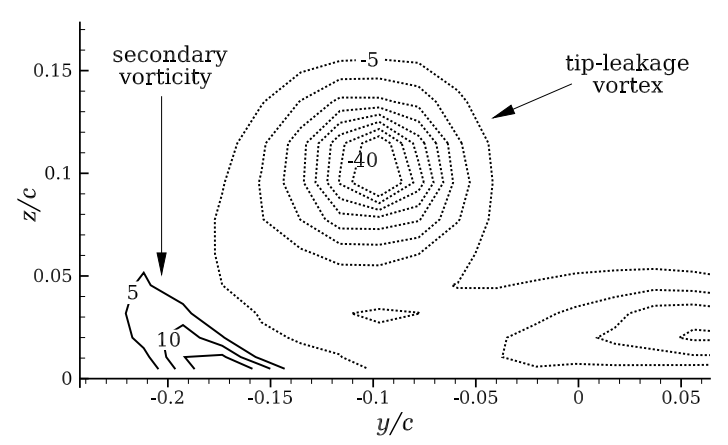

Fig. 10: Visualization of the tip leakage vortex flow with the secondary vorticity at the wall. The plot shows iso-lines of streamwise vorticity at $x / c=1.05$ for $h / c=$ $3.5 \%$. Negative vorticity is featured by dashed lines. Negative vorticity relates to the tip leakage vortex while positive vorticity relates to the secondary vortex

\section{Tip vortex trajectory}

The several measurement planes recorded with LDV allow tracing the locus of the vortex $\boldsymbol{x}_{c}$, which are shown with closed symbols in figure 11 . The locus is obtained by taking the centroid of axial vorticity in the tip-leakage vortex, that is

$\boldsymbol{x}_{c}=\frac{\int_{\Omega_{Q}} \omega_{x} \boldsymbol{x} d y d z}{\int_{\Omega_{Q}} \omega_{x} d y d z}$

where $\omega_{x}$ is the streamwise vorticity and $\Omega_{Q}$ is the zone corresponding to the tip vortex, here identified using positive values of the $Q$ criterion given by

$Q=\frac{1}{2}\left(\Omega_{i j} \Omega_{i j}-S_{i j} S_{i j}\right)$

with $\Omega_{i j}$ the vorticity tensor defined as

$\Omega_{i j}=\frac{1}{2}\left(\frac{\partial u_{i}}{\partial x_{j}}-\frac{\partial u_{j}}{\partial x_{i}}\right)$

The area $\Omega_{Q}$ is defined by $Q>0$ (Wu et al., 2007), here calculated based on the LDV data. In some cases LDV measurements were not possible in the vortex core due to irregular seeding and/or laser lighting, therefore the vorticity centroid could not be computed. In 
these cases the position of the tip vortex is estimated by locating the center of curvature of the pseudostreamlines surrounding the vortex core (see open symbols in figure 11), where LDV measurements remain accessible. Pseudo-streamlines are the apparent streamlines formed by the transverse flow, i.e. not accounting for the axial flow component.

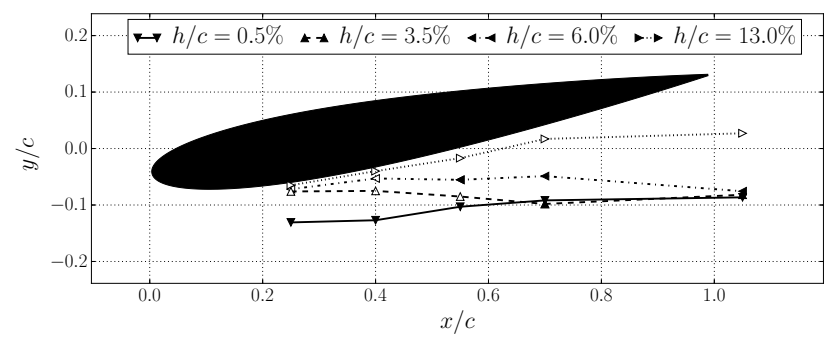

Fig. 11: Vortex center trajectory for a selection of gap heights. The vortex centers are obtained using the LDV measurements made at several stations along the blade. Closed symbols indicate the centroid of streamwise vorticity. Open symbols indicate the center of curvature of the streamlines which is used as a proxy to locate vortex center when LDV data inside the vortex core is not available

This ensemble of vortex locus allows to draw the trajectory of the tip leakage vortex, as presented in figure 11. The tip vortex develops differently depending on $h / c$. As previously observed in the literature (Flachsbart, 1931; Lakshminarayana and Horlock, 1963; Doukelis et al., 1998b), the tip-leakage vortex stays close to the blade for large gap to chord ratios, the separation of the vortex from the blade occurring at approximately $50 \%$ of chord for $h / c=13 \%$, while it evolves away from the blade at low ratios. In any case the tip vortex eventually moves away from the blade surface, which is opposite to what happens for an isolated wing, and is the consequence of the image effect of the casing wall. Notwithstanding the no-slip boundary condition at the casing wall, from an inviscid point of view, the effect of the wall upon the tip vortex is accounted for by an opposite vortex mirror of the primary one about the casing wall. This image vortex induces a transverse momentum away from the blade, that can usefully be referred as drift.

The effect of gap height $h / c$ and incoming boundary layer thickness on the vortex lateral and vertical positions are plotted in figures 12 and 13 respectively. Figure 12 shows a non monotonous evolution of lateral position, with a positive slope for $h / c<2 \%$ and a negative one for $h / c>4.5 \%$. These two opposing trends are

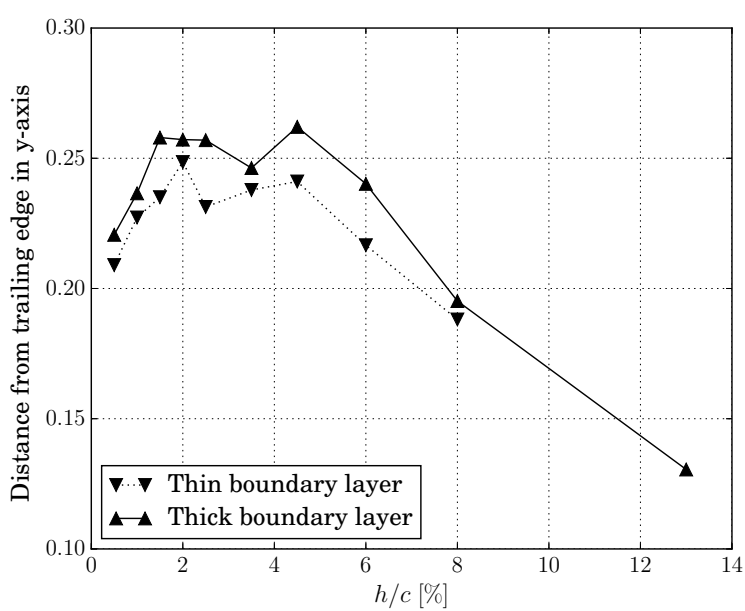

Fig. 12: Evolution of the vortex center lateral position, expressed as distance from blade trailing edge, as a function of gap height, at $x / c=1.05$. The vortex center position is obtained from the 5 -hole pressure probe measurements



Fig. 13: Evolution of the vortex center vertical position as a function of gap height, at $x / c=1.05$. The vortex center position is obtained from the 5 -hole pressure probe measurements

the consequence of the conflicting effects of delayed vortex detachment from the blade at larger gap ratios and increased drift effect at lower gap ratios due to closer wall proximity and accentuated effect of the image vorticity. A small and a large gap regime can also be seen in figure 13 for the vortex vertical position. At very large gap to chord ratios $h / c$, the tip-leakage vortex is expected to be similar to a wingtip vortex, meaning that the distance between the blade tip and the vortex center $\Delta z_{0}$ is not impacted by gap height anymore. This is tantamount to saying that $z_{v}=h+\Delta z_{0}$, where $\Delta z_{0}$ is a 
constant. This large gap regime seems to occur for gap heights larger than $8 \%$ of chord. At low gap to chord ratios, here for $h / c<3.5 \%$, the vertical position of the tip vortex also increases linearly, although more rapidly than gap height. For gap heights between 3.5\% and $8 \%$ of chord, the vortex vertical position transitions from the small gap trend to the large gap one.

Figures 12 and 13 show little effect of the boundary layer thickness on the vertical position and only a slight effect on the transverse position. A thicker incoming boundary layer allows an increased lateral position of the tip vortex, which is consistent with the numerical analysis of Brandt et al. (2002). This effect can be explained, at least for small gap heights, by considering the jet in cross-flow dynamics of the tip flow. The penetration of a jet in a cross-flow is driven by the ratio $R=U_{j}^{2} / U_{0}^{2}$, where $U_{0}$ is the main stream velocity and $U_{j}$ the jet exit velocity (Fearn and Weston, 1974). Based on the approach of Rains (1954), the gap exit velocity $U_{j}$ is essentially driven by the pressure distribution across the gap, which is not impacted by the incoming boundary layer thickness. Therefore a thicker boundary layer reduces $U_{0}$ at the gap exit without changing $U_{j}$, which increases the ratio $R$, provoking a larger push of the jet and a more detached formation of the tip vortex.

From the perspective of a compressor, and considering that an increased lateral position of the tip vortex is detrimental regarding surge margin, improvement is to be obtained by reducing the boundary layer thickness at the casing. Compressors typically feature gap to chord ratios smaller than $3 \%$, which corresponds to the first type of behavior just described. Then, both an increased gap to chord ratio and incoming boundary layer thickness lead to an increased lateral position of the tip vortex and thus may reduce the surge margin.

\section{Tip vortex circulation}

The determination of the tip leakage circulation is accomplished by first distinguishing the tip-leakage vortex from the secondary vorticity, as the two are present, see figure 10. Circulations $\Gamma$ and $\Gamma$ " corresponding to that of the tip-leakage vortex and of the secondary vortex, respectively, are hence introduced,

$\Gamma=\iint_{\omega_{x}<0} \omega_{x} d S \quad \Gamma^{\prime \prime}=\iint_{\omega_{x}>0} \omega_{x} d S$

In equation (5), the integration areas are shaped by the sign of the vorticity. To verify the accuracy of the circulation computed as the sum of streamwise vorticity, the total circulation $\Gamma_{t o t}=\Gamma+\Gamma$ " obtained with relation (5) is compared to the circulation of velocity in a closed contour $\mathcal{C}$, i.e.

$\Gamma_{t o t}=\oint_{\mathcal{C}} \mathbf{u} \cdot \mathbf{d l}$

The deviation between these two approaches to compute the total circulation $\Gamma_{\text {tot }}$ is below $2 \%$. Hence the values of circulation obtained from the sum of streamwise vorticity (5) have a similar accuracy as the one computed from the line integral (6).

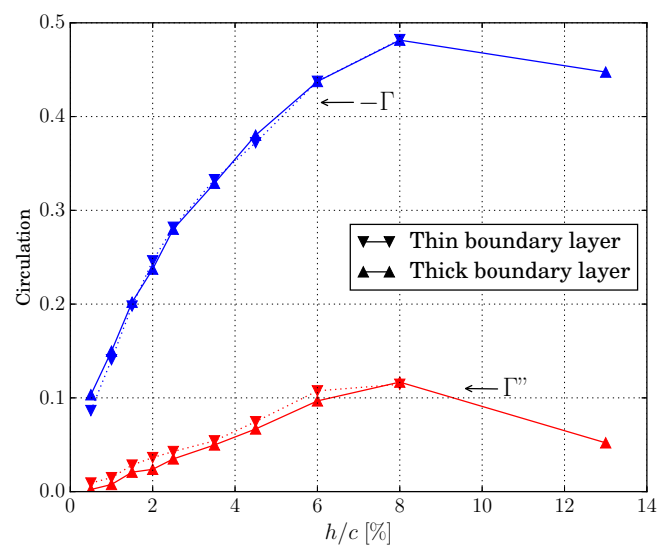

Fig. 14: Evolution of tip-leakage vortex circulation and secondary vorticity as a function of gap height at $x / c=$ 1.05 for the two values of the boundary layer thickness. The data is infered from the measurements performed with the 5-hole pressure probe

The evolutions of $\Gamma$ and $\Gamma$ " as a function of gap height $h / c$ are displayed in figure 14 for the two boundary layer thicknesses at the casing. These circulations increase with gap height up to $h / c=8 \%$ and are not affected by the incoming boundary layer thickness. Then, up to $h / c=13 \%$, a case only available for the thick incoming boundary layer configuration, a decrease occurs. The increase in the tip-leakage vortex circulation accounts for the growth of leakage flow with larger $h / c$. In return stronger tip vorticity results in reinforced secondary vorticity. For gap heights larger than $8 \%$ of chord, the decrease in tip-leakage vortex circulation can be explained by a reduction of blade lift induced by a combination of vortex downwash and reduced blade span.

\section{Axial velocity in the tip-leakage vortex}

At the vortex center, where in first order approximation $u_{y}, u_{z} \simeq 0$, Bernoulli theorem states that the axial velocity is mostly determined by the difference between 


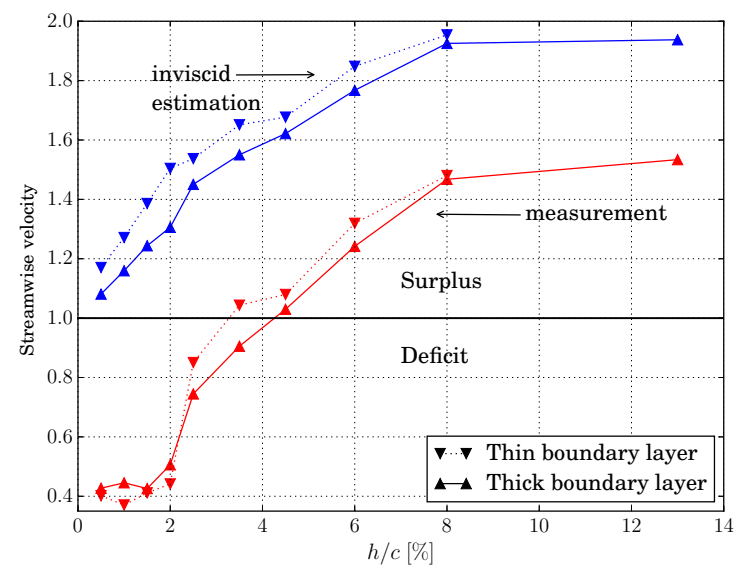

Fig. 15: Evolution of the streamwise velocity at the vortex center as a function of gap height as measured by the 5 -hole probe measurements at $x / c=1.05$ and as estimated by the Bernoulli evaluation in (7) considering zero total pressure loss in the vortex core

the static pressure drop $\Delta P_{s}=P_{s \infty}-P_{s}$ and the total pressure loss $\Delta P_{t}$, following

$\frac{u_{x}}{U_{\infty}}=\left(1+\frac{\Delta P_{s}}{\frac{1}{2} \rho U_{\infty}^{2}}-\frac{\Delta P_{t}}{\frac{1}{2} \rho U_{\infty}^{2}}\right)^{\frac{1}{2}}$

Figure 15 compares the measured value of $u_{x}$ at the vortex center to the inviscid estimate given by $(7)$ considering $\Delta P_{t}=0$, for varying gap height $h / c$. The axial velocity increases monotonously with $h / c$. The flow features a velocity deficit in the vortex core for low $h / c$ and a jet flow above $h / c=4 \%$. The effect of a thicker incoming boundary layer is to decrease the axial velocity. The inviscid estimate provides the good slope of the increase in $u_{x}$ while lying well above the experimental $u_{x}$ because of the presence of total pressure losses $\left(\Delta P_{t}>0\right)$ in the vortex core. Hence the increase in $u_{x}$ with gap height comes from the pressure drop at the vortex center, as the consequence of the strengthening of the tip-leakage vortex.

\section{Losses in the tip region}

Based on the theoretical analysis of Denton (1993), the increment of entropy will be used as the signature of losses in the flow. Indeed, in a compressor, the increase in entropy essentially comes from irreversible processes, since heat transfers can generally be neglected. Moreover, entropy is independent of the reference frame, therefore, from that perspective, the analysis performed on the present isolated and fixed blade can be extrapolated to a real compressor.

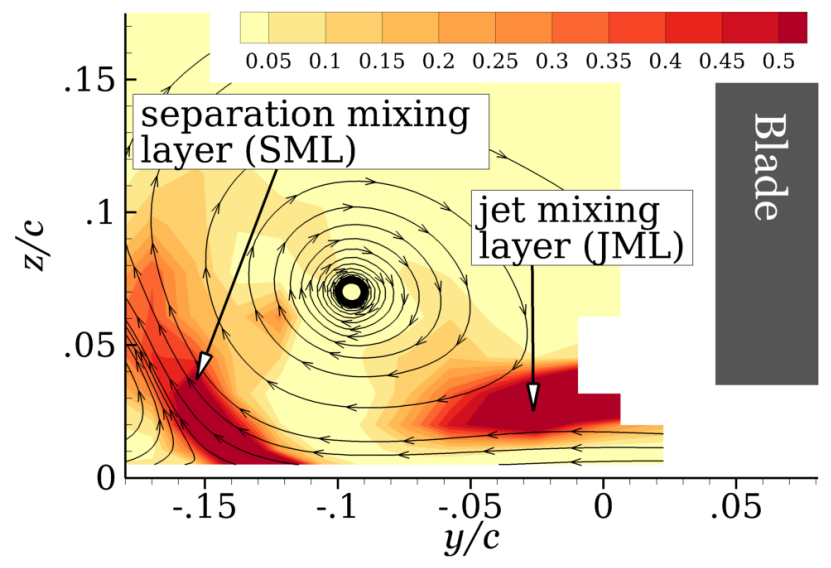

(a) $x / c=0.70$

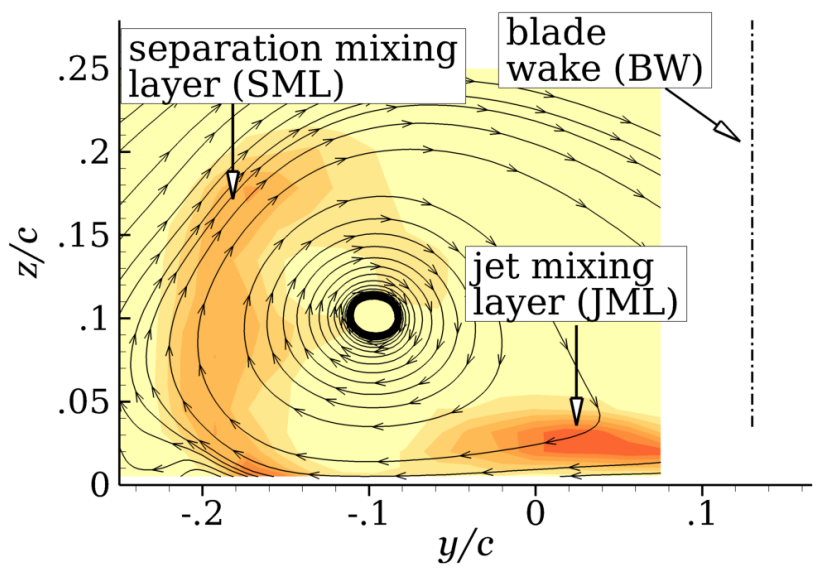

(b) $x / c=1.05$

Fig. 16: Distribution of mean flow entropy creation rate $\dot{s}_{m}$ and pseudo-streamlines of the transverse flow in the tip-leakage vortex area for $h / c=3.5 \%$ and thick incoming boundary layer

\section{Local distribution of losses}

Areas where losses occur can be identified by looking at where a particle of fluid undergoes an entropy increase, i.e. where the mean flow entropy production rate per unit of mass $\dot{s}_{m}$ is positive. For a steady and incompressible flow, it is possible to calculate this quantity using LDV measurements following

$\dot{s}_{m}=\frac{1}{T_{t \infty}}(2 \nu S_{i j} S_{i j}+\underbrace{\tau_{i j} \frac{\partial u_{i}}{\partial x_{j}}}_{P_{k}})$

This relationship is demonstrated in appendix A. The quantities $S_{i j}$ and $\tau_{i j}=-<u_{i}^{\prime} u_{j}^{\prime}>$ are the strain rate tensor and the Reynolds stress tensor, respectively. Equation (8) states that the entropy increases in areas of turbulent kinetic energy production $\left(P_{k}\right)$ and viscous dissipation $\left(2 \nu S_{i j} S_{i j}\right)$. Derivatives with respect to 




(a) $h / c=0.5 \%$

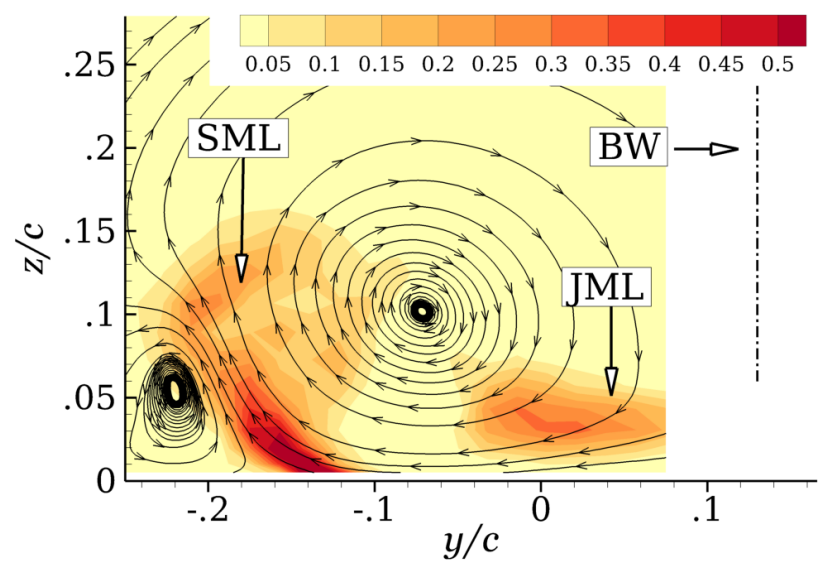

(b) $h / c=6.0 \%$



(c) $h / c=13.0 \%$

Fig. 17: Effect of gap height on the mean flow entropy creation rate $\dot{s}_{m}$ shown in transverse plane at $x / c=$ 1.05 , for the thick incoming boundary layer case. The plots include pseudo-streamlines of the transverse flow to locate the different structures $y$ and $z$ are about three orders of magnitude larger than those with respect to $x$. Hence $x$ derivatives in equation (8) are discarded.

The distribution of $\dot{s}_{m}$ in the tip-leakage vortex area is shown in figure 16 , for $h / c=3.5 \%$. Figure 16a shows that entropy is created in the mixing layer formed by the jet flow (JML) and in an area named separation mixing layer (SML), at the left of the tip-leakage vortex. The SML comes from the separation of the boundary layer at the casing wall, induced by the tip-leakage vortex. Downstream of the blade, it can be seen in figure $16 \mathrm{~b}$ that the magnitude of the losses in the jet mixing layer (JML) and separation mixing layer (SML) decreases along $x$ between stations $x / c=0.70$ and 1.05 . Note that entropy is also created in the blade wake (BW), however this area was not investigated with LDV measurements for this gap height.

The effect of gap height $h / c$ on the distribution of $\dot{s}_{m}$ is shown in figure 17. Except for $h / c=13 \%$, the blade wake (BW) is outside the measurement plane and is represented by the dash-dot line. For $h / c=0.5 \%$ (figure 17a), it seems that a significant part of the entropy is created in the separation mixing layer (SML). In the case $h / c=6.0 \%$ (figure $17 \mathrm{~b}$ ), the entropy production in the jet mixing layer (JML) and in the separation mixing layer (SML) have comparable magnitudes. The case $h / c=13 \%$, the largest gap, is eventually shown in figure $17 \mathrm{c}$. In this situation, no jet is present and the entropy is mostly created in the blade wake (BW) that rolls-up around the tip-leakage vortex.

To sum up, entropy production is not only found in the jet mixing layer, as previously observed by Storer and Cumpsty (1994), but also in the wake and in a mixing layer coming from the separation of the boundary layer at the casing wall induced by the tip-leakage vortex.

\section{Volume integrated losses}

The global loss $\mathcal{L}$ is considered to be the mass-flow averaged entropy increase per unit of mass occurring upstream of the $(y, z)$ plane $S_{0}$, such as

$\mathcal{L}=\frac{1}{\dot{m}} \iiint_{\Sigma} \rho \dot{s}_{m} d V$

In equation (9), $\dot{m}$ is the mass-flow going through $\Sigma$, the volume defined by the envelop of the path of fluid particles, or streamtube, taken far upstream, where the flow is uniform, and getting to the plane $S_{0}$ after flowing through the gap. 
It is shown in appendix $\mathrm{B}$ that $\mathcal{L}$ is related to the total pressure losses such as

$\mathcal{L}=\frac{1}{2} \frac{U_{\infty}^{2}}{T_{t \infty}} C_{P t}^{m}$

where

$C_{P t}^{m}=\frac{\iint_{S_{0}} C_{P t} u_{x} d S}{\iint_{S_{0}} u_{x} d S}$

is the mass-flow averaged total pressure loss coefficient considering a $(y, z)$-integration plane, which is defined with the pressure loss coefficient

$C_{P t}=\frac{P_{t \infty}-P_{t}}{\frac{1}{2} \rho U_{\infty}^{2}}$

The quantity $C_{P t}^{m}$ evaluates the global effects of the dissipative phenomena that occur upstream of the $S_{0}$ plane. At this plane $S_{0}$, located at $x / c=1.05$, and defined by $y / c \in[-0.35,0.15]$ and $z / c \in[0.015,0.325]$, measurements are carried out using the 5 -hole pressure probe.

To differentiate the contributions of transverse (tipleakage vortex) and streamwise (wake) flow components in the total pressure losses, $C_{P t}$ is decomposed as the sum of $C_{\text {vortex }}$ and $C_{\text {wake }}$, defined as

$$
C_{P t}=\underbrace{\left[\frac{P_{s \infty}-P_{s}}{\frac{1}{2} \rho U_{\infty}^{2}}-\frac{\left(u_{y}^{2}+u_{z}^{2}\right)}{U_{\infty}^{2}}\right]}_{C_{\text {vortex }}}+\underbrace{\left[1-\left(\frac{u_{x}}{U_{\infty}}\right)^{2}\right]}_{C_{\text {wake }}}
$$

Maps of $C_{\text {vortex }}$ and $C_{\text {wake }}$ are shown in figure 18 , for $h / c=3.5 \%$ and a thick incoming boundary layer. The coefficient $C_{\text {wake }}$ is high in the blade wake, in the boundary layer and in the streamwise velocity deficit area of the core of the tip-leakage vortex. This coefficient relates to the concept of blockage used by turbomachine engineers and is here referred to as wake loss coefficient. As for $C_{\text {vortex }}$ figure 18 shows that it is concentrated in the tip-leakage vortex core with a maximum at the vortex center. This quantity related to the transverse flow induced by the vortex is referred as vortex loss coefficient.

Considering a Rankine vortex model of circulation $\Gamma$ and core radius $a$ for the tangential flow of the tipleakage vortex, it is shown in appendix $\mathrm{C}$ that $C_{\text {vortex }}$ depends upon the square of the circulation and vortex core radius. The radial distribution of $C_{\text {vortex }}$ for the Rankine vortex is plotted in figure 19. The maximum of $C_{\text {vortex }}$ occurs at the vortex center and is equal to zero outside of the vortex core. This is comparable to the experimental observations made in figure 18. The
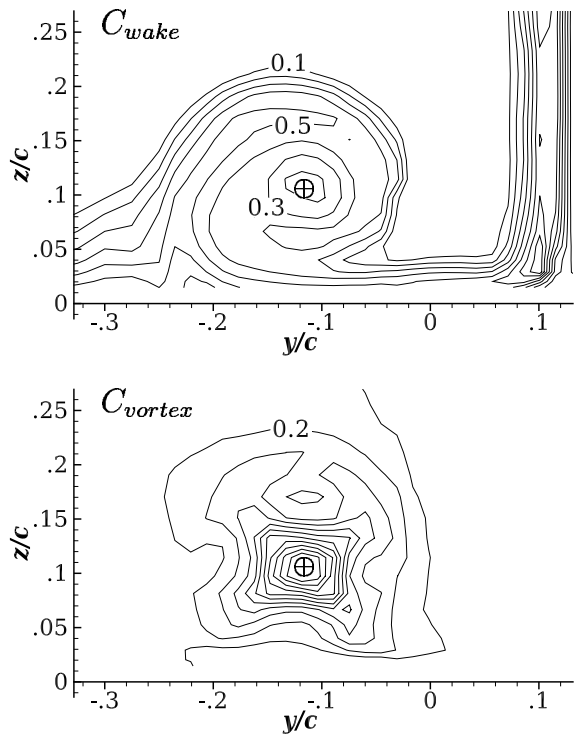

Fig. 18: Distribution of $C_{\text {wake }}$ and $C_{\text {vortex }}$ in a transverse plane at $x / c=1.05$, for $h / c=3.5 \%$ and the thick boundary layer case. The plot shows iso-contours of $C_{\text {wake }}$ and $C_{\text {vortex }}$ with increment of 0.1 between iso-lines. The symbol $\oplus$ indicates the position of the tip-leakage vortex center

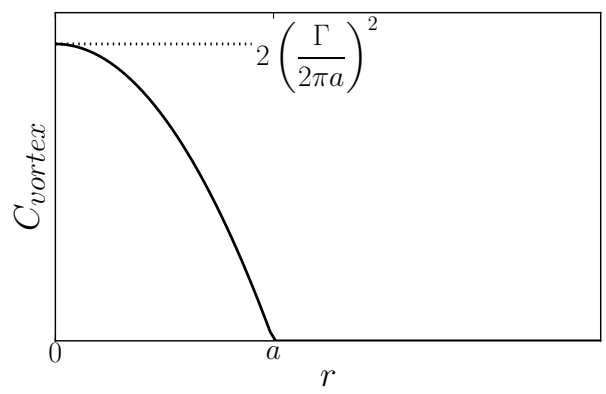

Fig. 19: Distribution of vortex loss coefficient $C_{\text {vortex }}$ in a Rankine vortex of circulation $\Gamma$ and core radius $a$

surface averaged vortex loss coefficient $C_{\text {vortex }}^{s}$, given by

$C_{\text {vortex }}^{s}=\frac{\iint_{S_{0}} C_{\text {vortex }} d S}{\iint_{S_{0}} d S}$

is independent of the core radius $a$, leaving the sole dependence on vortex circulation squared, that is

$C_{\text {vortex }}^{s}=\frac{A}{S_{0}} \Gamma^{2} \quad A=\frac{1}{4 \pi} \approx 0.080$

Figure 20 displays the experimental increment of averaged vortex loss $\Delta C_{\text {vortex }}^{s, m}$, either averaged upon mass flow rate (superscript ${ }^{m}$ ) or surface (superscript 
${ }^{s}$ ), against the tip-leakage vortex circulation squared $\Gamma^{2}$, where

$\Delta C_{\text {vortex }}^{s, m}=C_{\text {vortex }}^{s, m}-\left.C_{\text {vortex }}^{s, m}\right|_{h=0}$

with $\left.C_{\text {vortex }}^{s, m}\right|_{h=0}$ the vortex loss coefficient for a closed gap, which is extrapolated from the experimental data. It can be seen that the surface averaged increment of vortex loss $\Delta C_{\text {vortex }}^{\text {s }}$ (open symbols) follows a linear increase with $\Gamma^{2}$, in agreement with the Rankine approximation. The values of the linear coefficient $A$ are also close, with about 0.10 in the experiment and 0.08 in the model. The increase $\Delta C_{\text {vortex }}^{m}$ of the mass-flow average of $C_{\text {vortex }}$ (closed symbols) with $\Gamma^{2}$ is similar to the surface averaged one up to $\Gamma^{2}=0.18$. For high values of tip-leakage vortex circulation, i.e. $\Gamma^{2}>0.18$, the linear law slightly underestimates the mass-flow averaged $\Delta C_{\text {vortex }}^{m}$. This deviation corresponds to the appearance of a strong streamwise velocity surplus in the vortex core (see figure 15) that accentuates the contribution of this area, where $C_{\text {vortex }}$ is high, in the global count of mass-flow average of vortex losses.

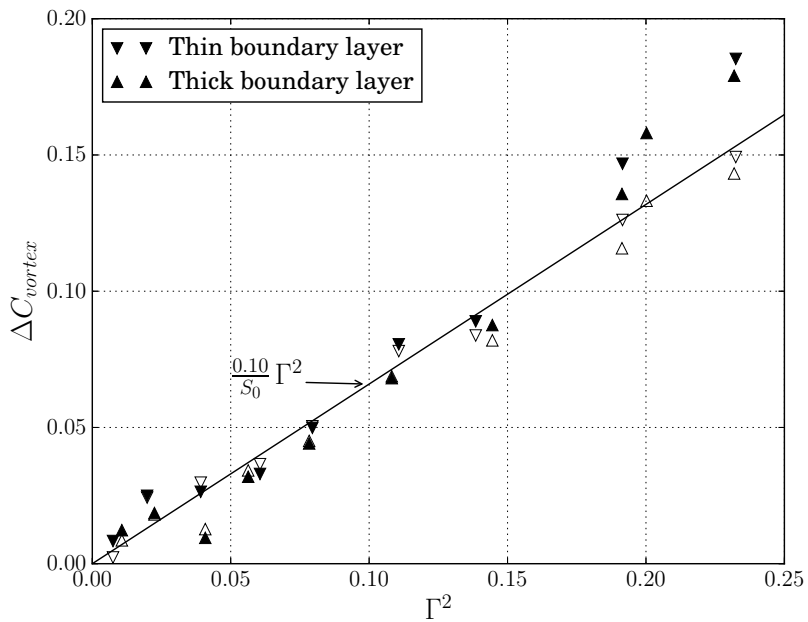

Fig. 20: Increment of vortex loss coefficient $\Delta C_{v o r t e x}^{s, m}$ with the tip-leakage vortex circulation squared at $x / c=$ 1.05 for the two values of the upcoming boundary layer thickness. Open symbols : surface average $\Delta C_{\text {vortex }}^{s}$. Closed symbols : mass-flow average $\Delta C_{\text {vortex }}^{m}$

Evolutions of $C_{P t}^{m}, C_{\text {vortex }}^{m}$ and $C_{\text {wake }}^{m}$ with gap height and incoming boundary layer thickness are presented in figure 21. It is observed that these coefficients all increase at small $h / c$ and then decrease, at different thresholds. The evolutions of $C_{P t}^{m}$ and $C_{\text {vortex }}^{m}$ follow similar trends, decreasing for $h / c>8 \%$ while $C_{\text {wake }}^{m}$ starts to decrease earlier, for $h / c>4 \%$. First, this shows that the increment of total pressure losses $\Delta C_{P t}^{m}$



Fig. 21: Evolution of mass-flow averaged loss coefficients $C_{P t}^{m}, C_{\text {vortex }}^{m}$ and $C_{\text {wake }}^{m}$ at $x / c=1.05$

with gap height is primarily driven by the vortex losses $C_{\text {vortex }}^{m}$. Second, the decrease in $C_{\text {wake }}^{m}$ induces a reduction of $C_{P t}^{m}$ increment with gap height for $h / c$ between $4 \%$ and $8 \%$ of chord. Third, the vortex losses $C_{\text {vortex }}^{m}$ are dominant in the total pressure losses $C_{P t}^{m}$ for intermediate and large gap configurations while $C_{\text {vortex }}^{m}$ and $C_{\text {wake }}^{m}$ count for almost the same at small gaps. The reduction of $C_{\text {vortex }}^{m}$ is the result of the decrease in tipleakage vortex circulation for $h / c>8 \%$, as shown in figure 14. Whereas the decrease in $C_{\text {wake }}^{m}$ for $h / c>4 \%$ can be attributed to the emergence of a streamwise velocity surplus in the tip-leakage vortex core (see figure 15).

The effect of the incoming boundary layer thickness is appreciated in figure 21. While no major difference is observed qualitatively, the trends being the same, the amplitudes of $C_{\text {vortex }}^{m}$ and especially $C_{\text {wake }}^{m}$ are affected, in opposite ways. A thinner boundary layer tends to appraise the contribution of $C_{\text {vortex }}^{m}$ compared to $C_{\text {wake. }}^{m}$. The value of $C_{P t}^{m}$ is also increased, by approximately 0.011 when the boundary layer is thicker. This points out the extra losses induced by a thicker boundary layer.

\section{Model for the vortex losses}

The objective here is to build a model to estimate the sensitivity of total pressure losses with gap height. The previous section showed that the increment of total pressure losses $\Delta C_{P t}^{m}$ with respect to gap height is essentially driven by the vortex losses $C_{\text {vortex }}^{m}$ and is weakly affected by the incoming boundary layer thick- 
ness, that is

$\Delta C_{P t}^{m} \simeq \Delta C_{\text {vortex }}^{m}$

Moreover the increment of vortex losses $\Delta C_{\text {vortex }}^{m}$, relative to the value at $h=0$, increases linearly with the tip-leakage vortex circulation $\Gamma$ squared, as shown in figure 20, and deviates slightly from this linear increase only for the highest values of circulation $\left(\Gamma^{2}>0.18\right)$. That is why the main focus here is to build a model for the tip-leakage circulation. The increment of vortex losses $\Delta C_{\text {vortex }}^{m}$ is then calculated with relation (15) with $A=0.1$. The model for the circulation is obtained by drawing an analogy between the tip-leakage flow and a jet in cross-flow, following our earlier analysis.

The jet in cross-flow produces a symmetrical counter-rotating vortex pair system which aligns with the direction of the cross-flow (Fearn and Weston, 1974; Jacquin, 1994; Karagozian, 2014). In the current analogy, the vortex pair consists of the tip-leakage vortex and the vortex generated by the symmetrical blade with respect to the casing wall as sketched in figure 22. A slip boundary condition is considered at the casing wall, meaning that the present model does not take into account the effect of the boundary layer. This is coherent with the observation of the weak influence of the incoming boundary layer thickness on the tip-leakage vortex circulation (see figure 14).

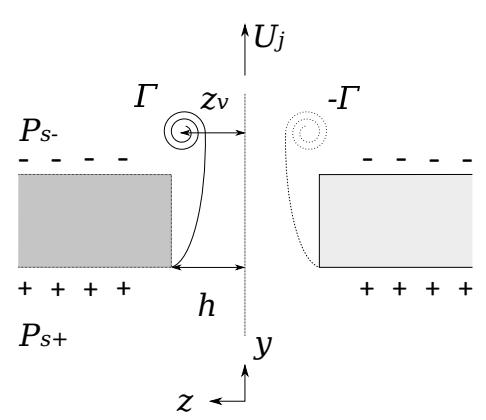

Fig. 22: Slotted wing configuration used to model the flow through the gap in an inviscid approximation, showing the subsequent roll-up of the jet flow outside the slot into two counter rotating vortices

\section{Description of the model}

The circulation of the rolling-up jet has been assessed by Jacquin (1994) using an analogy with tip vortices in the wake of an isolated finite wing. In this approach, this equivalent wing is modelled as a lifting line of span equal to the distance between the centers of the two vortices generated by the jet in cross-flow, i.e. $2 z_{v}$, with lift
$L$. Next the lift is obtained from the Kutta-Joukowski theorem as

$L=2 \rho U_{\infty} \Gamma z_{v}$

Then, the jet is assumed to be adapted and its thrust $T$ is assimilated to the lift $L$ of this equivalent wing such as

$T=\rho \overline{U_{j}^{2}} A_{j}=2 \rho U_{\infty} \Gamma z_{v}$

where $A_{j}$ represents the slot area (equal to $2 h c$ ) and $\overline{U_{j}^{2}}$ is the mean value of the jet velocity squared. Equation (19) can be conveniently reorganized as

$\frac{\Gamma}{c U_{\infty}}=\frac{h}{z_{v}} \frac{\overline{U_{j}^{2}}}{U_{\infty}^{2}}$

which yields the circulation $\Gamma$ in terms of gap height $h$, distance $z_{v}$ between the vortex center and the casing wall, and jet velocity $U_{j}$ at the gap exit. The latter can be related to the blade loading $C_{L}$ by considering that the flow entering the gap is driven by the pressure difference between the pressure and suction sides. As mentioned earlier this approach previously adopted by Rains (1954) is justified by LDV measurements showing the flow accelerating primarily in a plane transverse to the main flow (see figure $7 \mathrm{~b}$ ). At a given chordwise position $\xi$ one thus has

$\frac{U_{j}(\xi)}{U_{\infty}}=\chi_{D} \sqrt{\frac{P_{s+}(\xi)-P_{s-}(\xi)}{\frac{1}{2} \rho U_{\infty}^{2}}}$

which can be derived from Bernoulli theorem upon inserting an overall discharge coefficient $\chi_{D}$ to account for the losses in the gap. Then taking the square of relation (21) and its mean value leads to

$\frac{\overline{U_{j}^{2}}}{U_{\infty}^{2}}=\chi_{D}^{2} \underbrace{\int_{0}^{c} \frac{P_{s+}(\xi)-P_{s-}(\xi)}{\frac{1}{2} \rho U_{\infty}^{2} c} \mathrm{~d} \xi}_{\approx C_{L}}$,

where the mean pressure difference along the chord is assumed to be close to the airfoil lift coefficient $C_{L}$. Finally, replacing $\overline{U_{j}^{2}}$ in equation (20) by the expression (22) leads to

$\Gamma=\frac{h}{z_{v}} \chi_{D}^{2} C_{L}$

The circulation $\Gamma$ is normalized by the chord $c$ and the velocity $U_{\infty}$.

The increment of vortex losses is then estimated by inserting relation (23) in (15) and taking $A=0.1$, which leads to

$\Delta C_{\text {vortex }}^{m}=\frac{0.1}{S_{0}}\left(\frac{h}{z_{v}}\right)^{2} \chi_{D}^{4} C_{L}^{2}$ 


\section{Experimental validation}

The airfoil lift coefficient $C_{L}$ is obtained from pressure measurement at mid-span of the wing, and equals 1.01. The vertical position $z_{v}$ of the tip-leakage vortex center is described as a piecewise affine function of gap height

$$
\frac{z_{v}}{c}= \begin{cases}1.51\left(\frac{h}{c}\right)+0.05 & \text { for } \frac{h}{c} \leq 3.5 \% \\ 0.104 & \text { for } 3.5 \% \leq \frac{h}{c} \leq 8.5 \% \\ \frac{h}{c}+0.02 & \text { for } \frac{h}{c} \geq 8.5 \%\end{cases}
$$

which fits the experimental data provided in figure 13 .

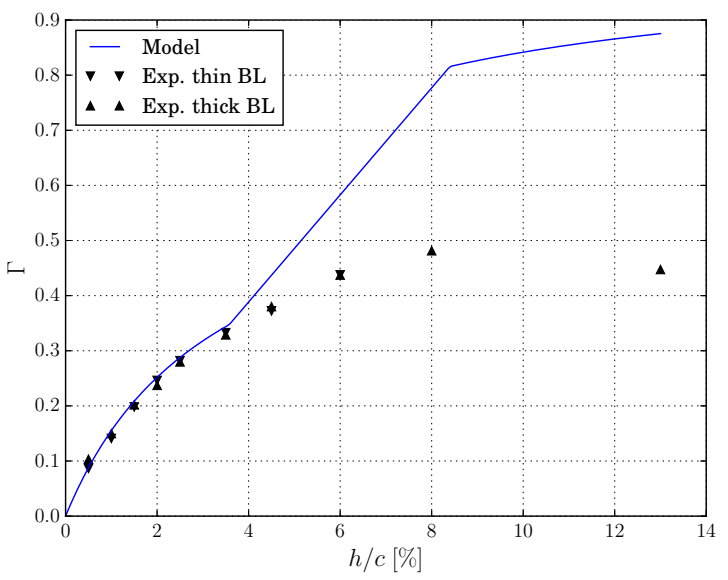

Fig. 23: Comparison of the circulation $\Gamma$ provided by the model (straight line) against experimental data (symbols) for the two values of the upcoming boundary layer thickness, as a function of gap height

In figure 23, the predictions of this model are compared to the tip-leakage vortex circulation $\Gamma$ obtained in the experiments by integrating the negative vorticity only. For gap heights smaller than $3.5 \%$ of the chord, a good agreement with experimental data is obtained. Note that the discharge coefficient $\chi_{D}$ is set to 1. The overestimation of the circulation by the model for $h / c>3.5 \%$ suggests that the analogy with a jet in cross-flow is not valid anymore for larger gaps. In practice, for $h / c<3.5 \%$, relation (23) and (24) become respectively

$\Gamma=\frac{h / c}{a_{1}\left(\frac{h}{c}\right)+a_{0}} \chi_{D}^{2} C_{L}$

and

$\Delta C_{\text {vortex }}^{m}=\frac{0.1}{S_{0}}\left(\frac{h / c}{a_{1}\left(\frac{h}{c}\right)+a_{0}}\right)^{2} \chi_{D}^{4} C_{L}^{2}$

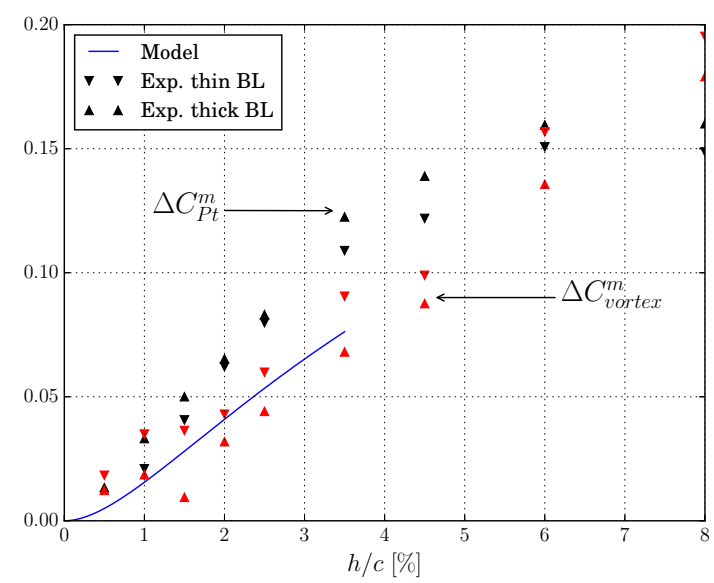

Fig. 24: Increment of total and vortex loss as a function of gap height, for the two values of the upcoming boundary layer thickness. Comparison between the model (straight line) and experimental data (symbols)

where $a_{1}$ and $a_{0}$ are empirical coefficients, here found equal to 1.51 and 0.05 respectively.

In figure 24 , the modelled $\Delta C_{\text {vortex }}^{m}$ is compared to experimental data, from which the extrapolated values at $h=0$ are subtracted. This approach gives a good prediction of the sensitivity of $C_{\text {vortex }}^{m}$ to gap height, which allows to capture the main part of the increment of $C_{P t}^{m}$. To get a better prediction of the increment of total pressure loss with gap height, the wake loss coefficient $C_{\text {wake }}^{m}$ would have to be taken into account.

Note that the fitting coefficients for $z_{v}$ in relation (25), i.e. $a_{0}$ and $a_{1}$ in equations (26) and (27), may depend on $x$ and on the blade loading. Indeed, for a jet in cross-flow, the vortex spacing depends not only on $x$ but also on jet exit velocity $U_{j}$ (Fearn and Weston, 1974). This suggests that $z_{v}$ depends on $x$ and $U_{j}$, itself linked to the blade loading through relation $(22)$.

The value 1 of the discharge coefficient $\chi_{D}$ adopted in this model is larger than the value of 0.8 found in literature (Rains, 1954; Moore and Tilton, 1988; Storer and Cumpsty, 1994). This higher value of $\chi_{D}$ may compensate the larger values of $z_{v}$ caused by the vortex rebound, that comes from the interaction of the tipleakage vortex with the no-slip casing wall in the experiment (Barker and Crow (1977)).

\section{Conclusions}

The sensitivity of the tip-leakage flow to gap height and the incoming boundary layer thickness has been investigated using an isolated and fixed blade setup. Detailed measurements, using LDV, a 5-hole pressure probe and 
pressure taps, were conducted in a low-speed wind tunnel for a Reynolds number of 550000 . It appears that the tip-leakage flow features are primarily impacted by the gap height. The effects of this parameter on the flow can be divided into three regimes : a small gap regime for gap heights smaller than $3.5 \%$ of chord, a large gap regime for gap larger than $8 \%$ of chord and a intermediate regime in-between. Increasing the gap height moves the maximum of transverse velocity in the gap, or minimum of pressure, toward the blade trailing edge. Moreover, the tip-leakage vortex remains attached to the blade over a longer portion of chord and its circulation increases when the gap height is enlarged. This also tends to reduce the streamwise velocity defect, present at the vortex center for small gaps, leading to the apparition of a streamwise velocity excess for gaps larger than $4 \%$ of chord. In front of the blade, the flow is found to separate if gap height $h$ is smaller than the momentum thickness $\theta^{*}$ and if blade loading is high, leading to the formation of a horseshoe vortex. A thicker incoming boundary layer at the casing tends to move the tip-leakage vortex away from the blade, whereas the static pressure distribution at the casing wall and the tip-leakage vortex circulation, are not impacted by the incoming boundary layer thickness.

A careful analysis of the losses in the tip region has been realized. Local losses are defined by the entropy production rate, which has been computed from LDV measurements. At the gap exit, entropy is created not only in the jet mixing layer but also in a mixing layer associated with the separation of the casing boundary layer induced by the tip-leakage vortex. Overall losses are reflected by total pressure losses, which have been decomposed in two terms, one representing the contribution of the areas of streamwise velocity deficit and identified as a wake loss, and the other one, identified as a vortex loss, that evolves with the tip-leakage vortex circulation squared. The evolution of total pressure losses with gap height is primarily driven by the vortex loss coefficient. A thicker incoming boundary layer decreases the vortex losses and increases the wake losses, which globally leads to higher total pressure losses, without altering significantly their evolution with the gap height.

Finally to describe the increment in total pressure losses impaired in the gap, the tip-leakage flow has been modelled as a jet in cross-flow. This model gives a good estimation of the tip-leakage vortex circulation for gaps smaller than $3.5 \%$ of the chord. The tip vortex circulation is a proxy to evaluate the increment in pressure loss, on the basis of the net influence of the vortex loss coefficient, which depends on the circulation squared.
The range of gap height accounted for by the model is valid for most turbomachinery applications.

Acknowledgements The present work is supported by the ANR project NumERICCS (ANR-15-CE06-0009), led by Pr Georges Gerolymos. The 2D RANS calculation has been performed in the framework of the elsA three-party agreement between AIRBUS, SAFRAN and ONERA which are co-owners of this software. The authors would like to thank Pr Jacques Borée and Pr Georges Gerolymos who have shared their expertise on theoretical aspects regarding the entropy production in the flow.

\section{References}

Barker SJ, Crow SC (1977) The motion of twodimensional vortex pairs in a ground effect. Journal of Fluid Mechanics 82(4):659-671

Bloxham M, Bons J, Hollis R (2008) Horseshoe vortex control with leading edge endwall boundary layer removal. In: 4th Flow Control Conference, p 4319

Borello D, Hanjalic K, Rispoli F (2007) Computation of tip-leakage flow in a linear compressor cascade with a second-moment turbulence closure. International Journal of Heat and Fluid Flow 28(4):587-601

Brandt H, Fottner L, Saathoff H, Stark U (2002) Effects of the inlet flow conditions on the tip clearance flow of an isolated compressor rotor. In: ASME Turbo Expo 2002: Power for Land, Sea, and Air, American Society of Mechanical Engineers, pp 1123-1132

Chassaing P (2010) Mécanique des Fluides : Éléments d'un premier parcours, 3rd edn. Cépaduès

Decaix J, Balarac G, Dreyer M, Farhat M, Münch C (2015) Rans and les computations of the tip-leakage vortex for different gap widths. Journal of Turbulence 16:309-341, DOI 10.1080/14685248.2014.984068

Denton J (1993) Loss mechanisms in turbomachines. In: ASME 1993 International Gas Turbine and Aeroengine Congress and Exposition, American Society of Mechanical Engineers, pp V002T14A001V002T14A001

Devenport WJ, Simpson RL (1990) Time-dependent and time-averaged turbulence structure near the nose of a wing-body junction. Journal of Fluid Mechanics 210:23-55

Doukelis A, Mathioudakis K, Papailiou K (1998a) The effect of tip clearance gap size and wall rotation on the performance of a high-speed annular compressor cascade. In: ASME 1998 International Gas Turbine and Aeroengine Congress and Exhibition, American Society of Mechanical Engineers

Doukelis A, Mathioudakis K, Papailiou K (1998b) Investigation of the 3 -d flow structure in a highspeed annular compressor cascade for tip clearance 
effects. In: ASME 1998 International Gas Turbine and Aeroengine Congress and Exhibition, American Society of Mechanical Engineers, pp V001T01A012V001T01A012

Fearn R, Weston RP (1974) Vorticity associated with a jet in a cross flow. AIAA Journal 12(12):1666-1671

Flachsbart O (1931) Spaltverluste an Tragflügeln. ZAMM-Journal of Applied Mathematics and Mechanics/Zeitschrift für Angewandte Mathematik und Mechanik 11(6):411-414

Hasan M, Casarella M, Rood E (1986) An experimental study of the flow and wall pressure field around a wing-body junction. Journal of vibration, acoustics, stress, and reliability in design 108(3):308-314

Heyes F, Hodson H, Dailey G (1992) The effect of blade tip geometry on the tip leakage flow in axial turbine cascades. Journal of Turbomachinery 114(3):643-651

$\mathrm{Hu} \mathrm{H}$, Yang Z (2008) An experimental study of the laminar flow separation on a low-reynolds-number airfoil. Journal of Fluids Engineering 130(5):051101

Inoue M, Kuroumaru M (1989) Structure of tip clearance flow in an isolated axial compressor rotor. Journal of Turbomachinery 111(3):250-256

Jacob M, Grilliat J, Camussi R, Caputi Gennaro G (2010) Aeroacoustic investigation of a single airfoil tip leakage flow. International Journal of Aeroacoustics

Jacquin L (1994) Phenomenological description and simplified modelling of the vortex wake issuing from a jet in a cross flow. La Recherche Aérospatiale 2:117133

Julien HL, Kays W, Moffat R (1969) The turbulent boundary layer on a porous plate-an experimental study of the effects of a favorable pressure gradient. Technical Report HMT-4, Thermosciences Division, Department of Mechanical Engineering, Stanford University

Kameier F, Neise W (1997) Experimental study of tip clearance losses and noise in axial turbomachines and their reduction. Journal of Turbomachinery 119(3):460-471

Kang S, Hirsch C (1993) Experimental study on the three-dimensional flow within a compressor cascade with tip clearance: Part Ivelocity and pressure fields. Journal of Turbomachinery 115(3):435-443

Kang S, Hirsch C (1996) Numerical simulation of threedimensional viscous flow in a linear compressor cascade with tip clearance. Journal of Turbomachinery 118(3):492-502

Karagozian AR (2014) The jet in crossflow. Physics of Fluids 26(10):101303

Lakshminarayana B, Horlock JH (1963) Tip-clearance flow and losses for an isolated compressor blade.
Technical Report ARC-R/M-3316, Aeronautical Research Council London (England)

Menter FR (1994) Two-equation eddy-viscosity turbulence models for engineering applications. AIAA Journal 32(8):1598-1605

Millikan CB (2018) Aerodynamics of the Airplane. Courier Dover Publications

Moore J, Tilton J (1988) Tip leakage flow in a linear turbine cascade. Journal of Turbomachinery 110(1):18-26

Muthanna C, Devenport W (2004) Wake of a compressor cascade with tip gap, part 1: Mean flow and turbulence structure. AIAA Journal 42(11):2320-2331

Pope SB (2011) Turbulent flows. Cambridge Univ. Press, Cambridge

Praisner T, Smith C (2006) The dynamics of the horseshoe vortex and associated endwall heat transfer. Part I: Temporal behavior. Journal of Turbomachinery 128(4):747-754

Prandtl L (1919) Tragflügeltheorie. II. Mitteilung. Nachrichten von der Gesellschaft der Wissenschaften zu Göttingen, Mathematisch-Physikalische Klasse pp 107-137

Rains DA (1954) Tip clearance flow in axial compressors and pumps. PhD thesis, California Institute of Technology

Saathoff H (2001) Rotor-Spaltströmungen in Axialverdichtern. PhD thesis, Technische Universität Braunschweig

Saathoff H, Stark U (2000) Tip clearance flow induced endwall boundary layer separation in a single-stage axial-flow low-speed compressor. 2000-GT, p 0501

Smith G, Cumpsty NA (1982) Flow phenomena in compressor casing treatment. Tech. Rep. CUED/ATurbo/TR 112, Whittle Laboratory, Department of Engineering, University of Cambridge

Storer J, Cumpsty N (1991) Tip leakage flow in axial compressors. Journal of Turbomachinery 113(2):252 259

Storer J, Cumpsty N (1994) An approximate analysis and prediction method for tip clearance loss in axial compressors. Journal of Turbomachinery 116(4):648656

Vo HD, Tan CS, Greitzer EM (2008) Criteria for spike initiated rotating stall. Journal of Turbomachinery 130(1):011023

Wu JZ, Ma HY, Zhou MD (2007) Vorticity and vortex dynamics. Springer Science \& Business Media

Yakhot A, Anor T, Liu H, Nikitin N (2006) Direct numerical simulation of turbulent flow around a wallmounted cube: spatio-temporal evolution of largescale vortices. Journal of Fluid Mechanics 566:1-9 


\section{Appendix}

\section{Entropy creation rate for the mean flow}

The first objective of this section is to clarify the the definition of the entropy production rate for the mean flow. The second objective is to demonstrate that this entropy production rate can be estimated using velocity measurements from LDV. This work generalizes the analysis carried out by Denton (1993) on the relation between entropy increment and viscous forces. The following assumptions are considered:

1. The fluid is a perfect gas : $P=\rho R T$

2. The flow is in a steady state and incompressible $: \operatorname{div}(\mathbf{u})=$ 0 and $M \ll 1$ (for the present case $M=0.1$ )

3. Variations of total pressure and temperature are small, i.e. $\left|P_{t}-P_{t \infty}\right| \ll P_{t \infty}$ and $\left|T_{t}-T_{t \infty}\right| \ll T_{t \infty}$ (in the present experiment $\left.\left|P_{t}-P_{t \infty}\right|<10^{-2} \times P_{t \infty}\right)$

4. Heat transfers are neglected

Starting with Gibbs-Duhem relation one can relate entropy variation to total enthalpy and pressure for the instantaneous field following

$T_{t} d s=d h_{t}-R T_{t} \frac{d P_{t}}{P_{t}}$.

Assuming that $\left|P_{t}-P_{t \infty}\right| \ll P_{t \infty}$ and $\left|T_{t}-T_{t \infty}\right| \ll T_{t \infty}$ we have

$T_{t \infty} d s=d h_{t}-\underbrace{\frac{R T_{t \infty}}{P_{t \infty}}}_{1 / \rho_{t \infty}} d P_{t}$.

Insofar as the Mach number is small $(M=0.1)$, we assume that $\rho \approx \rho_{t \infty}$, which leads to

$T_{t \infty} \frac{d s}{d t}=\frac{d h_{t}}{d t}-\frac{1}{\rho} \frac{d P_{t}}{d t} \quad$ where $\quad \frac{d \bullet}{d t}=\boldsymbol{\bullet}=\frac{\partial \bullet}{\partial t}+u_{i} \frac{\partial \bullet}{\partial x_{i}}$

Then the Reynolds decomposition is applied to equation (30), considering the assumptions of a steady and incompressible flow, which leads to

$T_{t \infty} \dot{s}_{m}=<u_{i}>\frac{\partial}{\partial x_{i}}<h_{t}>-\frac{1}{\rho}<u_{i}>\frac{\partial}{\partial x_{i}}<P_{t}>$,

with $<\bullet>$ the Reynolds average and with $\dot{s}_{m}$ defined as

$$
\begin{aligned}
\dot{s}_{m}= & <u_{i}>\frac{\partial}{\partial x_{i}}<s>+\frac{\partial}{\partial x_{i}}<u_{i}^{\prime} s^{\prime}> \\
& -\frac{1}{T_{t \infty}} \frac{\partial}{\partial x_{i}}<u_{i}^{\prime} e^{\prime}>.
\end{aligned}
$$

In (33), $e^{\prime}$ represents the fluctuation of internal energy per unit of mass. This quantity $\dot{s}_{m}$ can be understood as the equivalent rate of entropy production for the mean flow, which is not the same as the mean entropy production rate $\dot{s}$. From now on, the symbol $\langle\bullet\rangle$ is dropped for mean flow quantities in order to reduce the amount of notation. Equation (31) thus becomes

$T_{t \infty} \dot{s}_{m}=u_{i} \frac{\partial h_{t}}{\partial x_{i}}-\frac{1}{\rho} u_{i} \frac{\partial P_{t}}{\partial x_{i}}$.

To replace the right hand side of equation (33), let's consider the RANS energy equation

$$
\begin{aligned}
u_{j} \frac{\partial h_{t}}{\partial x_{j}}= & \left(2 \nu S_{i j}+\tau_{i j}\right) \frac{\partial u_{i}}{\partial x_{j}}+u_{i} \frac{\partial}{\partial x_{j}}\left(2 \nu S_{i j}+\tau_{i j}\right) \\
& -\frac{1}{\rho} \frac{\partial q_{j}}{\partial x_{j}} \quad,
\end{aligned}
$$

and the RANS mean flow kinetic energy equation

$\frac{1}{\rho} u_{i} \frac{\partial P_{t}}{\partial x_{i}}=u_{i} \frac{\partial}{\partial x_{j}}\left(2 \nu S_{i j}+\tau_{i j}\right)$

with $S_{i j}$ the strain rate tensor, $\tau_{i j}=-<u_{i}^{\prime} u_{j}^{\prime}>$ the Reynolds stress tensor and $q_{j}$ the heat flux. Inserting relations (34) and (35) in equation (33) leads to

$T_{t \infty} \dot{s}_{m}=\left(2 \nu S_{i j}+\tau_{i j}\right) \frac{\partial u_{i}}{\partial x_{j}}-\frac{1}{\rho} \frac{\partial q_{j}}{\partial x_{j}}$,

which is similar to the relation obtained by Chassaing (2010). This relation can also be written as follows

$T_{t \infty} \dot{s}_{m}=2 \nu S_{i j} S_{i j}+P_{k}-\frac{1}{\rho} \frac{\partial q_{j}}{\partial x_{j}}$,

with

$P_{k}=\tau_{i j} \frac{\partial u_{i}}{\partial x_{j}}$

the production of turbulent kinetic energy. Equation (37) shows that the equivalent mean flow entropy production rate $\dot{s}_{m}$ corresponds to the sum of viscous dissipation $\left(2 \nu S_{i j} S_{i j}\right)$, production of turbulent kinetic energy $\left(P_{k}\right)$ and heat power $(\operatorname{div}(\mathbf{q}))$. Upon assuming that the heat power is negligible, equation (37) yields

$\dot{s}_{m}=\frac{1}{T_{t \infty}}\left(2 \nu S_{i j} S_{i j}+P_{k}\right)$.

This relation only depend on mean flow velocity gradients and on the Reynolds stress tensor, therefore it is possible to compute $\dot{s}_{m}$ from LDV measurements.

\section{Averaged total pressure losses and en- tropy creation rate}

The objective of this section is to demonstrate the equivalence between mass-flow average total pressure losses $C_{P t}^{m}$ and the volume integrated rate of entropy production $\rho \dot{s}_{m}$ in a streamtube. The start is relation (33), which, taking into account that the flow is incompressible, leads to

$T_{t \infty} \rho \dot{s}_{m}=\operatorname{div}\left(\mathbf{u} \cdot \rho h_{t}\right)-\operatorname{div}\left(\mathbf{u} \cdot P_{t}\right)$.

This relation is integrated over the streamtube $\Sigma$ composed of an inlet boundary $S_{i n}$, an outlet boundary $S_{0}$ and a lateral boundary $S_{l a t}$. The inlet boundary is taken upstream of the blade where flow and thermodynamic conditions are homogeneous. The lateral boundary $S_{\text {lat }}$ is characterized by $\mathbf{u} . \mathbf{n}=0$, where $\mathbf{n}$ is the local normal vector. Then the divergence theorem applied to the integral of (40) yields

$$
\begin{aligned}
T_{t \infty} \iiint_{\Sigma} \rho \dot{s}_{m} d V & =\iint_{S_{i n}} P_{t \infty} U_{\infty}\left(\mathbf{e}_{x} \cdot \mathbf{n}\right) d S \\
& -\iint_{S_{0}} P_{t}(\mathbf{u} \cdot \mathbf{n}) d S+\Delta H_{t},
\end{aligned}
$$

with

$$
\Delta H_{t}=\iint_{S_{0}} \rho h_{t}(\mathbf{u . n}) d S-\iint_{S_{i n}} \rho h_{t \infty} U_{\infty}\left(\mathbf{e}_{x} \cdot \mathbf{n}\right) d S .
$$

In the present study we consider a fixed setup, i.e. no mechanical power is exchanged. Therefore, assuming no heat transfer 
at the boundaries of the streamtube $\Sigma$, we have $\Delta H_{t}=0$. Moreover, mass-flow being conserved between $S_{i n}$ and $S_{0}$, equation (41) becomes

$T_{t \infty} \iiint_{\Sigma} \rho \dot{s}_{m} d V=\iint_{S_{0}}\left(P_{t \infty}-P_{t}\right)(\mathbf{u} \cdot \mathbf{n}) d S$

The mass-flow averaged total pressure losses $C_{P t}^{m}$ is then introduced in equation (43), with

$C_{P t}^{m}=\frac{1}{\dot{m}} \iint_{S_{0}} C_{P_{t}} \rho(\mathbf{u . n}) d S$,

where $\dot{m}$ represents the mass flow through the streamtube $\Sigma$, and with

$C_{P t}=\frac{P_{t \infty}-P_{t}}{\frac{1}{2} \rho U_{\infty}^{2}}$,

leading to

$\iiint_{\Sigma} \rho \dot{s}_{m} d V=\frac{1}{2} \frac{U_{\infty}^{2}}{T_{t \infty}} \dot{m} C_{P t}^{m}$

The average entropy increase per unit of mass $\mathcal{L}$, defined as

$\mathcal{L}=\frac{1}{\dot{m}} \iiint_{\Sigma} \rho \dot{s}_{m} d V$

is introduced in relation (46), which gives

$\mathcal{L}=\frac{1}{2} \frac{U_{\infty}^{2}}{T_{t \infty}} C_{P t}^{m}$

Relation (48) states the equivalence between the mass-flow averaged total pressure losses and the average entropy increase per unit of mass.

\section{Steady axisymmetric vortex analysis}

This section establishes a relation between the vortex loss coefficient $C_{\text {vortex }}$, introduced in equation (13), and the circulation of the tip-leakage vortex. In a cylindrical reference frame $(x, r, \theta), C_{\text {vortex }}$ is defined by

$C_{\text {vortex }}=\frac{P_{s \infty}-P_{s}}{\frac{1}{2} \rho U_{\infty}^{2}}-\frac{u_{\theta}^{2}}{U_{\infty}^{2}}$

The radial velocity $u_{r}$ is taken equal to zero. An axisymmetric steady inviscid flow is in radial equilibrium, that is

$\frac{\partial P_{s}}{\partial r}=\rho \frac{u_{\theta}^{2}}{r}$

Upon introducing this relation in (49) leads to

$C_{\text {vortex }}=\frac{2}{U_{\infty}^{2}} \int_{r}^{\infty} \frac{u_{\theta}^{2}}{\xi} d \xi-\frac{u_{\theta}^{2}}{U_{\infty}^{2}}$.

Let us now consider the Rankine vortex, defined as

$u_{\theta}(r)=\left\{\begin{array}{l}\frac{\Gamma}{2 \pi a}\left(\frac{r}{a}\right), r<a \\ \frac{\Gamma}{2 \pi r}, r \geq a\end{array}\right.$, where $a$ is the vortex core radius and $\Gamma$ is the vortex circulation. Based on this model, the coefficient $C_{\text {vortex }}$ becomes

$C_{\text {vortex }}=\left\{\begin{array}{l}\frac{2}{U_{\infty}^{2}}\left(\frac{\Gamma}{2 \pi a}\right)^{2}\left[1-\left(\frac{r}{a}\right)^{2}\right], r<a \\ 0, r \geq a\end{array}\right.$.

which shows that the $C_{\text {vortex }}$ coefficient is maximum at the vortex center and is equal to zero outside the vortex core.

The surface average of $C_{v o r t e x}$ over a disk of radius $R$ is defined as

$C_{\text {vortex }}^{s}=\frac{1}{\pi R^{2}} \int_{0}^{R} C_{\text {vortex }}(r) \cdot 2 \pi r d r$

If the integration area includes the whole vortex core $(R>a)$, one has

$C_{\text {vortex }}^{s}=\frac{A}{S_{0}} \frac{\Gamma^{2}}{U_{\infty}^{2}}, S_{0}=\pi R^{2}, A=\frac{1}{4 \pi}, R>a$

Equation (55) shows that the surface average of $C_{v o r t e x}$ is proportional to the vortex circulation squared, divided by the integration area. This relation does not depend on the vortex core radius $a$. If $S_{0}$ and $\Gamma$ are normalized by the velocity $U_{\infty}$ and a reference length, relation (55) eventually becomes

$C_{\text {vortex }}^{s}=\frac{A}{S_{0}} \Gamma^{2}$. 